MgII observations using the MSFC Solar Ultraviolet Magnetograph

Edward West ${ }^{1}$, Jonathan Cirtain ${ }^{1}$, Ken Kobayashi, ${ }^{2}$ John Davis ${ }^{2}$ and Allen Gary ${ }^{2}$

${ }^{1}$ NASA/MSFC, ${ }^{2}$ UAH

This paper will describe the scientific goals of our sounding rocket program, the Solar Ultraviolet Magnetograph Investigation (SUMI). This paper will present a brief description of the optics that were developed to meet SUMI's scientific goals, discuss the spectral, spatial and polarization characteristics of SUMI's optics, describe SUMI's flight which was launched $7 / 30 / 2010$, and discuss what we have learned from that flight.

SPIE Solar Physics and Space Weather Instrumentation III

Conference OP404

San Diego, CA

$8 / 21-25 / 11$ 


\title{
MgII observations using the MSFC Solar Ultraviolet Magnetograph
}

\author{
Edward West ${ }^{\text {a }}$, Jonathan Cirtain ${ }^{\text {a }}$, Ken Kobayashi ${ }^{\text {b }}$, John Davis ${ }^{\text {, }}$, \\ Allen Gary ${ }^{b}$ and Mitzi Adams ${ }^{a}$ \\ ${ }^{a}$ Marshall Space Flight Center/NASA, 320 Sparkman Dr., Huntsville, AL 35805-1912
${ }^{b}$ The University of Alabama in Huntsville, 320 Sparkman Dr., Huntsville, AL 35805-1912
}

\begin{abstract}
This paper will describe the scientific goals of our sounding rocket program, the Solar Ultraviolet Magnetograph Investigation (SUMI). This paper will present a brief description of the optics that were developed to meet SUMI's scientific goals, discuss the spectral, spatial and polarization characteristics of SUMI's optics, describe SUMI's flight which was launched 7/30/2010, and discuss what we have learned from that flight.
\end{abstract}

Keywords: MgII polarization, VUV, SUMI, Solar Ultraviolet Magnetograph, VUV spectrograph, sounding rocket.

\section{INTRODUCTION}

This section will describe the science goals $(\S 1.1)$ of the Marshall Space Flight Center (MSFC) Solar Ultraviolet Magnetograph (SUMI), describe the instrument with emphasis on the MgII observations that were made (§1.2) and summarize the observations that were obtained during SUMI's first flight (§1.3).

\subsection{Science goals}

SUMI's goal is to measure the magnetic field in the upper chromosphere/lower transition region of the Sun's atmosphere. The reason to make those measurements is given in the following paragraphs:

The problem of how energy, stored in the solar magnetic field, is released to heat the corona and drive the dynamic phenomena of the outer atmosphere, flares and coronal mass ejections, remains unsolved. Neither theoretical or numerical models nor current observations are able to provide a conclusive explanation. A substantial fraction of this energy propagates outward into the interplanetary medium in the form of electromagnetic (EM) radiation, energetic particles, and the solar wind that creates, controls and modifies the structure of the interplanetary medium. Currently space weather effects orbiting satellites, and in the future, these will be a major concern for astronauts working on the lunar surface and traveling through interplanetary space. The solar atmosphere dynamics are the initial conditions for any model that describes and predicts space weather. Therefore understanding the processes that contribute to both the steady and transient release of magnetic energy into the solar atmosphere is a crucial element of these studies.

To understand the fundamental processes that lead to the explosive release of magnetic energy in solar flares and that initiates and propels coronal mass ejections (CMEs), the magnetic field needs to be observed and its topology and evolution inferred prior to and during the energy release process . Success has been achieved in the characterization of the full vector magnetic field in the photosphere, where $\beta$, the ratio of the gas pressure to the magnetic pressure, is $\geq 1$. At higher levels in the atmosphere (Figure 1) ${ }^{1}$ where $\beta<1$, the magnetic field, through the Lorentz force, controls the structure and dynamics of the solar plasma, and rapid changes in its structure can produce the observed energetic events. However, observations of the magnetic field at these higher levels have proven to be difficult, placing a serious limitation on our understanding of the physical processes

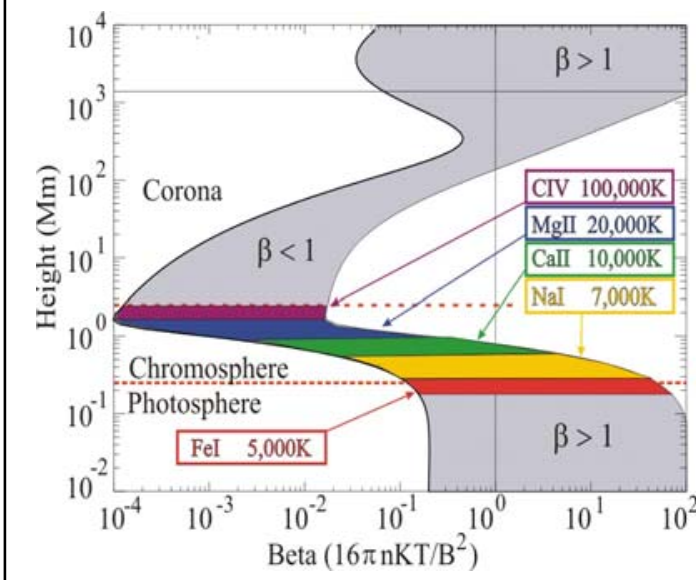

Figure 1. Ratio of gas pressure to magnetic pressure $(\beta)$ as a function of height, for an assumed range of photospheric magnetic field strength of 100 to $2500 \mathrm{G}^{1}$. 
occurring there.

The missing element is the ability to understand and describe quantitatively the transition layer where the field is no longer pressure-dominated as in the photosphere. The need to understand the energy release processes was identified two decades $\mathrm{ago}^{2}$, and was reaffirmed as the major conclusion of a workshop on the definition of the scientific goals for "Beyond Solar-B" ${ }^{3}$. To understand this process, full vector magnetic field measurements at several different heights in the solar atmosphere, from the photosphere to the upper transition region where the field becomes nearly force free, will be required. The vector field in the photosphere and low chromosphere has been measured with ground-based instruments. Space-based instruments such as the Michelson Doppler Interferometer (MDI) on the Solar \& Heliospheric Observatory (SoHO) have demonstrated that continuous, distortion free, observations of the longitudinal magnetic field from space are very important in our understanding of the Sun's magnetic field. The Solar Optical Telescope (SOT) on Hinode and the Helioseismic and Magnetic Imager (HMI) on the Solar Dynamics Observatory (SDO) spacecraft are now extending our space based observations to include photospheric vector magnetic fields. Although these high resolution observations will greatly advance our knowledge of the behavior of the magnetic field in the photosphere, they shed little information on how the magnetic field higher in the solar atmosphere transitions to a nearly force-free state. This requires measurement of the field using spectral lines that are formed at temperatures above $20,000{ }^{\circ} \mathrm{K}$. The two spectral lines chosen for SUMI are strong resonance pairs of MgII at $280 \mathrm{~nm}$ and CIV at $155 \mathrm{~nm}$. Because these lines occur in the vacuum ultraviolet (VUV), they are inaccessible from the ground. Together with the FeI, NaI and CaII lines, they form a quintet of strong magnetically sensitive lines which can be used in future missions to spatially map the magnetic field in three dimensions. Such a future space mission is the Magnetic Transition Region Probe (MTRAP) ${ }^{4}$. One of the objectives for the Solar Ultraviolet Magnetograph Investigation (SUMI) is the demonstration that the MgII and CIV lines can meet the requirements for this force-free region.

Goal for the SUMI sounding rocket program: To make vector magnetic field measurements in the upper chromosphere and lower transition region.

The Ultraviolet Spectrometer and Polarimeter (UVSP) instrument on board the Solar Maximum Mission (SMM, 1980) was the first space-based instrument to try to map and understand polarization in the transition region. The UVSP observations of MgII suggested that this line was a potential candidate for magnetic field measurements in the upper chromosphere ${ }^{5}$. Although UVSP attempted measurements of the CIV line, poor photon statistics and the loss of the ability to observe the CIV lines early in the mission resulted in a failure to produce convincing evidence that longitudinal magnetic field measurements were possible. While the scientific value of these observations has been acknowledged ${ }^{6,7}$, the measurements are difficult because they are in the vacuum ultraviolet (VUV) wavelength range where the optical efficiency of standard optics is low. The SUMI sounding rocket program was born from several development programs ${ }^{8,9,10}$ to improve the overall wavelength and polarization efficiency for the CIV (155nm) measurement while including the MgII measurements in its observing program. While these development programs were successful, accommodating the dual-line instrument in a sounding rocket envelope has been challenging.

Measuring CIV has always been the driving force in the development of SUMI. This resonance line is formed in the relatively thin transition region which simplifies its interpretation, although the actual height of formation can be problematic. However, the most difficult instrumental problem is the weak line splitting in UV. As a result the magnetic sensitivity, $\lambda^{2} \mathrm{~g}$, where $\mathrm{g}$ is the Landé $\mathrm{g}$ factor and $\lambda$ is the wavelength, is low compared to lines in the visible and infrared. Table 1 compares the SUMI lines with lines that are being used in photospheric and low chromospheric magnetographs. Certainly the results from SUMI will impact future missions as scientist try to understand the 3-D structure of the Sun's magnetic field.

Table 1: This table shows a comparison of candidate lines that are being developed for current and future space-based photospheric, chromospheric and transition region vector magnetic field measurements.

\begin{tabular}{|c|c|c|c|c|c|}
\hline $\begin{array}{c}\text { Spectral } \\
\text { lines }\end{array}$ & $\begin{array}{c}\lambda \\
(\mathrm{nm})\end{array}$ & $\mathrm{g}$ & $\begin{array}{c}\lambda^{2} \mathrm{~g} \\
\mathrm{~mm}^{2}\end{array}$ & $\begin{array}{c}\text { Height } \\
(\mathrm{km})\end{array}$ & Remarks \\
\hline \multicolumn{8}{|c|}{ Chromosphere } \\
\hline CIV & $\mathbf{1 5 5 . 0}$ & $\mathbf{1 . 2}$ & $\mathbf{0 . 2 9}$ & $\mathbf{2 2 0 0}$ & SUMI \\
\hline MgII & $\mathbf{2 8 0 . 0}$ & $\mathbf{1 . 2}$ & $\mathbf{0 . 9 4}$ & $\mathbf{2 0 0 0}$ & SUMI \\
\hline CaII & $\mathbf{8 5 4 . 2}$ & $\mathbf{1 . 1}$ & $\mathbf{8 . 0 2}$ & $\mathbf{1 3 1 9}$ & \\
\hline NaI & $\mathbf{5 8 9 . 5}$ & 1.3 & 4.51 & 700 & \\
\hline \multicolumn{7}{|c|}{ Photosphere } \\
\hline FeI & $\mathbf{6 3 0 . 2}$ & $\mathbf{2 . 5}$ & $\mathbf{9 . 9 2}$ & $\mathbf{2 5 0}$ & Hinode \\
\hline
\end{tabular}

MTRAP $^{6}$ : The Magnetic Transition Region Probe has all of these lines in its baseline mission requirements. 


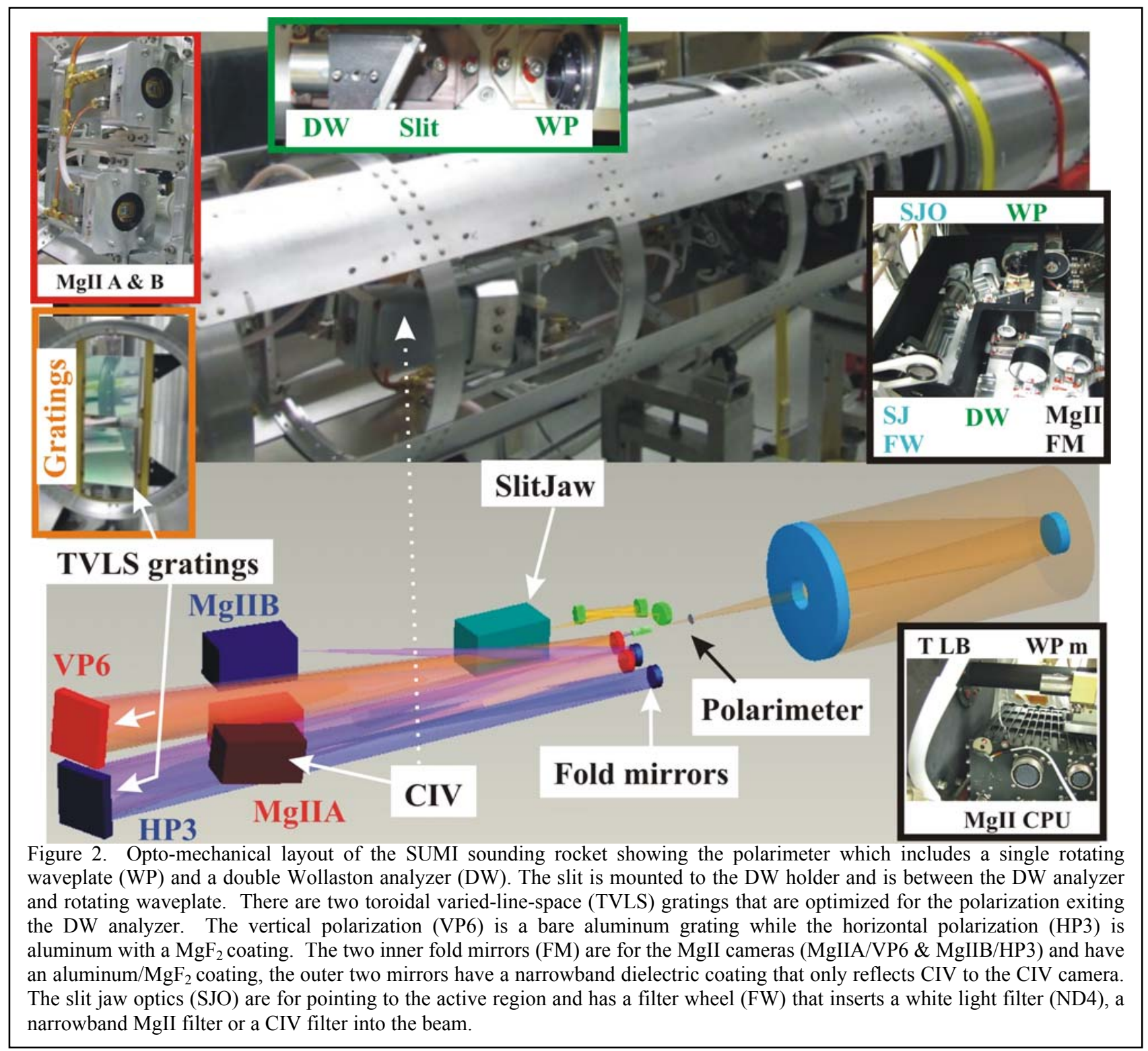

\subsection{Instrument description}

The instrument package includes a spectro-polarimeter for the scientific observations and a slitjaw camera system for pointing (white light images) and two "narrowband" filters to provide context images of the MgII (FWHM 40nm) and CIV (FWHM 30nm) lines. SUMI has been described in detail in several SPIE papers ${ }^{10,12}$.

In Figure 2 the optical model of SUMI is shown along with the mechanical components that determine the spectral and polarization resolution of the instrument. The SUMI telescope is an innovative "cold mirror" design that achieves its spectral filtering through the use of multilayer coatings. An important design requirement for solar telescopes is the rejection of the unwanted solar energy before it is concentrated onto the secondary. The standard solution is a Gregorian design that places the primary focus onto a field stop in front of the secondary mirror that rejects most of the energy at the expense of a reduced field of view. Gregorian telescopes are physically longer than Cassegrain or Ritchey-Chretien designs where the secondary mirror is placed between the primary and the telescope focus. Because our measurements are only interested in two narrow wavebands, we can use a "cold mirror" design to reject the unwanted energy. Customized dielectric high reflectivity (DHR) coatings are applied to both the primary and secondary. The dielectric coating reflects only the two spectral bands covering the CIV and MgII wavelengths. The measured out-of-band 
rejection of the coatings remains excellent across the optical and infrared wavelengths. The coatings for the MgII lines $(280 \mathrm{~nm})$ are deposited first. This coating was adjusted to minimize the reflectivity at $310 \mathrm{~nm}$ that would contaminate the observations of the $2^{\text {nd }}$ order CIV $(155 \mathrm{~nm})$ measurement. The CIV dielectric stack is then deposited on top of the MgII coating. This minimizes the absorption loss at CIV. The unwanted visible and infrared wavelengths incident on the primary mirror pass through the DHR coatings and fused silica mirror where they strike a spherically figured, aluminum coated back surface. This back surface reflects this incident radiation as a collimated beam out through the entrance aperture of the telescope into space. The front surface of the secondary has the same dielectric coatings to further reduce the out-of-band wavelengths ( $\sim 4 \%$ from primary mirror). To reduce the out-of-band light further, the back of the secondary mirror has an anti-reflection coating followed by a light-absorbing cone ${ }^{10}$.

Our Ritchey-Chretien design provides a more compact telescope which, within a fixed instrument length, increases the spectrograph length and, therefore, the spectral resolution. This design is an on-axis, radially symmetric telescope that minimizes instrumental polarization.

\subsubsection{Polarimeter Design}

The polarimeter consists of a rotating waveplate located in front of the spectrograph slit with a double Wollaston analyzer mounted behind the slit. Because of the low flux and weak linear polarization signal of the CIV emission line, our plan is to measure the circular polarization (longitudinal field) at CIV with exploratory linear polarization measurements to verify our radiometry calculations. For MgII, whose intensity is two orders of magnitude larger than $\mathrm{CIV}$, both linear and circular polarization measurements are possible.

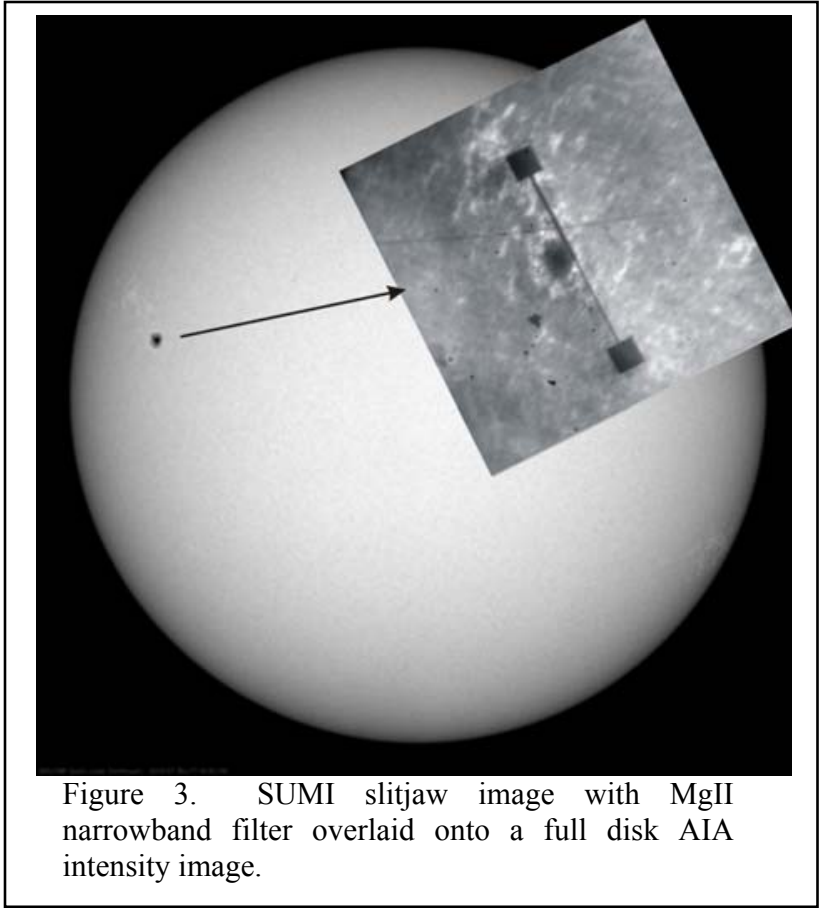

The waveplate is a zero order $\mathrm{MgF}_{2}$ design that is a "quarterwave" retarder at $\mathrm{CIV}\left(\sim 283^{\circ}\right)$ and a $\sim 138^{\circ}$ retarder at $\mathrm{MgII}^{12}$. The strength of the MgII line allows approximately one hundred polarization images to be acquired in the same time as a single CIV polarization map. Therefore, the circular polarization efficiency of the SUMI waveplate is maximized at $\sim 100 \%$ at CIV while still achieving $\sim 70 \%$ at $\mathrm{MgII}$. While the polarization efficiency does not affect the exposure time, which is dictated by photon statistics; it does affect the time required to obtain a given polarization resolution.

The SUMI analyzer is a double Wollaston polarizer that uses the birefringence of $\mathrm{MgF}_{2}$ to split the incident light into two linear polarization signals, thereby achieving both high broadband polarization efficiency and a total transmission greater than $50 \%$, even at $\mathrm{CIV}^{9}$.

\subsubsection{Spectrograph Design}

The SUMI spectrograph consists of two toroidal variedline-space (TVLS) gratings ${ }^{13,14}$, one for each linear polarization channel exiting the double Wollaston analyzer. Toroidal gratings were used to minimize the aberrations associated with the double Wollaston analyzer.

To optimize the instrument's imaging performance within a compact envelope, the spectrograph was designed to measure CIV in $2^{\text {nd }}$ order and MgII in $1^{\text {st }}$ order. The two TVLS gratings each have the same ruling variations as a function of position across their surface. This ruling variation differs by about $2 \%$ around the central groove density of 2440 lines per $\mathrm{mm}$. Both gratings have the same concave toroidal radii of curvature: $1500 \mathrm{~mm}$ in the dispersion axis and $1602 \mathrm{~mm}$ in the spatial direction. With this design, the spectrograph's wavelength resolution is significantly improved while minimizing reflection losses by avoiding additional optics that would be required by other spectrograph designs ${ }^{13}$.

Following the gratings, fold mirrors redirect the two polarization states of CIV and of MgII onto their own wavelength specific camera. The MgII fold mirrors are aluminum, while the CIV mirrors have the same CIV DHR coatings that are used on the telescope mirrors ${ }^{10}$. This reflection is an additional filter to reduce the $310 \mathrm{~nm}$ contamination of the CIV line. 


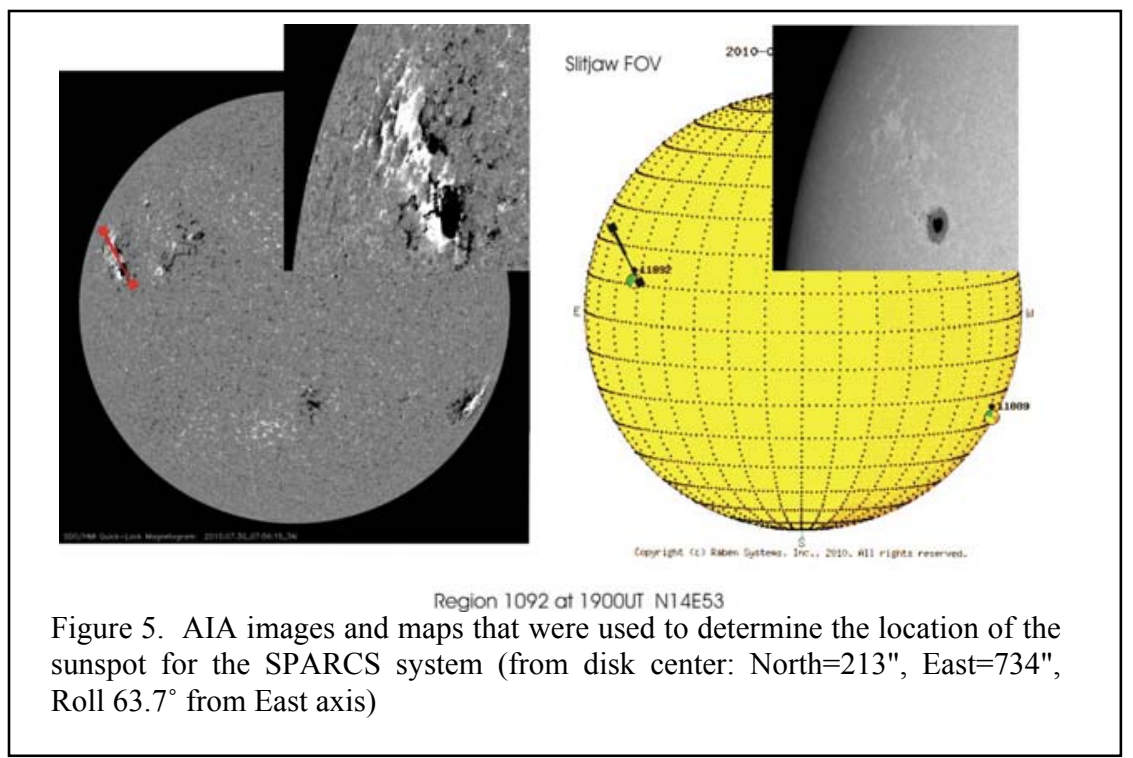

\subsubsection{The Slit and Slit-Jaw Camera}

SUMI's slit has a unique structure for our exploratory observations. The slit is cut into a $6 \mathrm{~mm}$ thick diamond turned aluminum substrate and is $\sim 3.7 \mathrm{~mm}$ long $(3.5$ arc minutes) with $0.5 \times 0.5 \mathrm{~mm}(30 \mathrm{arcsec})$ boxes on each end. The width of the slit has been split into two sections, a $30 \mu \mathrm{m}(\sim 1.6$ arc seconds) and a $60 \mu \mathrm{m}(\sim 3.2$ arc second). Due to the exploratory nature of the SUMI measurements, the uncertainty in the target at launch and the limited observing time of the flight $(\sim 6$ minutes), this structure was adopted to give SUMI a larger dynamic range and to capture enough photons to make meaningful polarization measurements at CIV.

The slit-jaw camera has three functions: 1) to enable the operator to place the spectrograph slit on the region of interest during target acquisition; 2) to record any jitter or drift in the pointing system during the flight; and 3) to provide context images for the grating scans after the target is acquired. This camera has a $5.6^{\prime} \times 5.6^{\prime}$ field of view. To improve the readout rate during target acquisition, the camera uses $2 \times 2$ on chip binning. The camera changes back into full resolution (binning 1x1) after the target is acquired.

\subsubsection{Spectrograph cameras}

The MgII, CIV and slitjaw cameras have the same optical characteristics. These E2V CCDs use a bare silicon structure (BN) to enhance their VUV sensitivity. When these cameras were ordered, the BN processing was only available in the $512 \times 512 \times 13 \mu \mathrm{m}$ pixel frame transfer format and a $1024 \times 256 \times 26 \mu \mathrm{m}$ full frame format. The $13 \mu \mathrm{m}$ pixel size corresponds to a plate scale for the MgII cameras of $20.3 \mathrm{~m} \AA$ and 0.46 arcsec per pixel (twice the TVLS resolution). The CIV camera (26 $\mu \mathrm{m}$ pixels) corresponds to a plate scale of $18.8 \mathrm{~m} \AA$ and 0.91 arcsec per pixel.

\subsection{Flight characteristics}

Even though the solar cycle was just coming out of solar minimum in the spring of 2010, we were fortunate that SUMI was launch (7/30/2010) just as Active Region 11092 (AR) emerged and rotated onto the disk. This region was a simple H-type sunspot that was an ideal candidate for relating the magnetic field in the photosphere to the magnetic field that SUMI wanted make. However, the location of the AR did not meet our original launch criteria (within 0.5 solar radius of disk center). With no launch window available the following week and a launch delay of at least two weeks (and no guarantee of a sunspot), the decision was made to continue with the launch. While converting SUMI's polarization measurements into magnetic fields would have been difficult due to the "large" optical depth of the CIV and MgII lines, this region would provide stronger linear polarization signals. Therefore, SUMI's MgII measurements, which measures the full Stokes vector, would benefit from these stronger linear polarization signals.

Our CIV radiometry calculations showed that linear polarization measurements were not possible for a sounding rocket's 6 minute observing time. While the location of the AR might help the MgII linear measurements, the strength of the CIV 
circular polarization signals would be weaker. Therefore, the location of the active region was less than optimum for CIV but was more than adequate for evaluating the engineering and technology performance of SUMI.

\section{DESCRIPTION OF OBSERVATIONS}

Because of programmatic requirements, SUMI was prepared for launch during the summer of 2008. Since SUMI requires an active region to make its magnetic field/polarization measurements that launch date slipped to 2009 and finally to July 30,2010 . This initial flight was an engineering flight: (1.) to verify that the cold mirror technology on SUMI's telescope mirrors could be used as a "prefilter" to isolate the wavelength bands for future solar telescope designs $^{10},(2$.) to show that simultaneous spectro-polarimetry measurements of orthogonal polarizations could be made on the two CIV emission lines (155.0 and $154.8 \mathrm{~nm}$ ) and the two MgII emission lines (279.8 and $280.4 \mathrm{~nm}$ ), (3.) to verify that the mechanical structure and fixtures could maintain the tight optical alignment tolerances, (4.) to show that the commercially-acquired cameras could survive a $10 \mathrm{G}$ launch, (5.) and to obtain images to check and adjust the radiometry calculations and optimize the exposure times in future flights.

Table 2 shows the observing program that the SUMI computers completed and the image files that were obtained during the flight. While the mission met its major engineering success criteria, there were two unexpected problems that limited the science for SUMI. This section will describe how the performance of the waveplate (\$2.1) allowed only MgII linear

Table 2. SUMI observing program that was completed during the 7/30/2010 flight. Exposures (exp) are in ms and the waveplate (WP) Fast Axis (FA) position in degrees. The launch was at 18:21:00 UT.

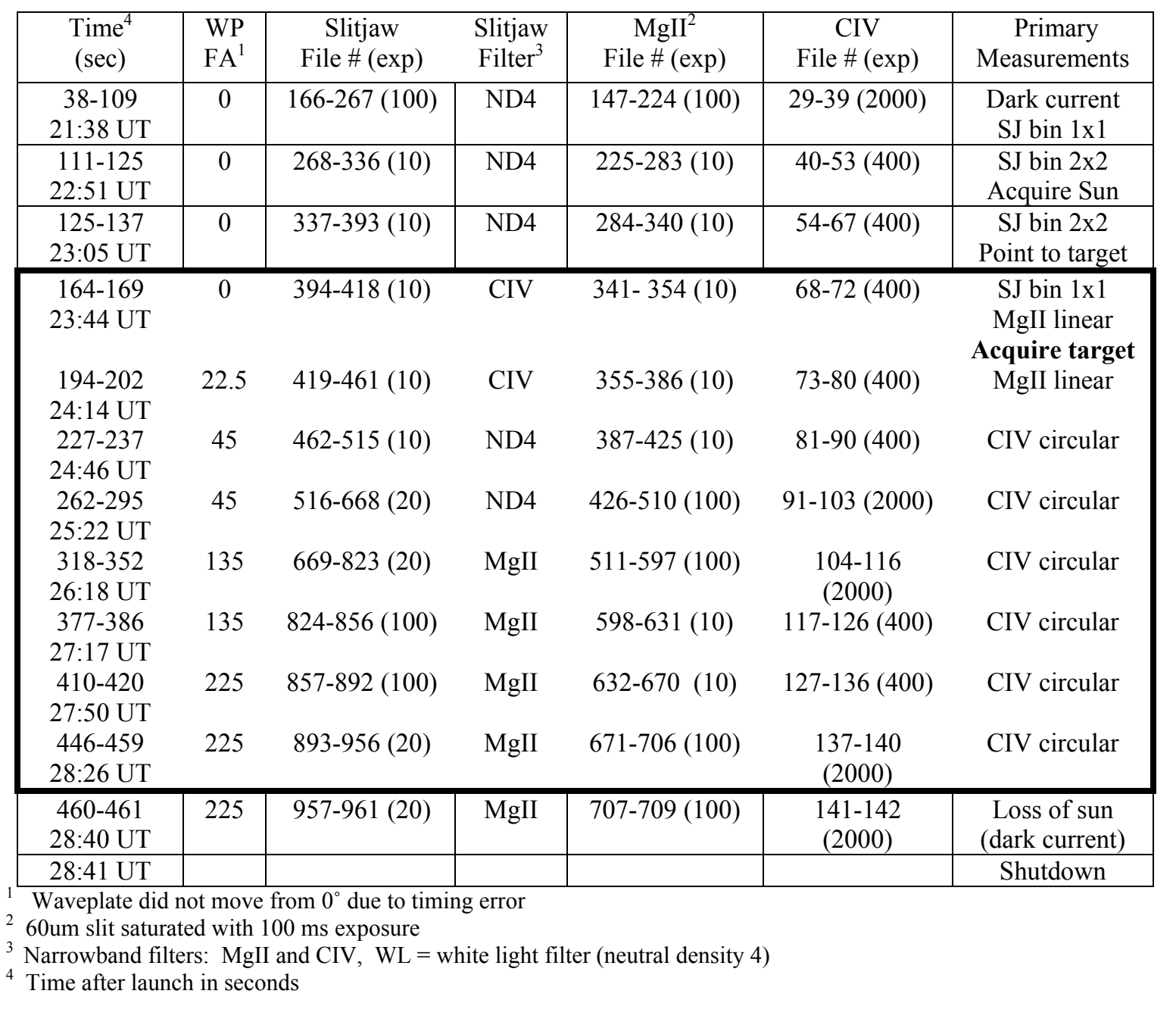


Table 3. Polarization Response to SUMI's rotating waveplate ( $\Delta=$ measured retardance, Kobayashi, 2005). With the waveplate rotation failure, the only measurements that were made were with the fast axis (FA) at $0^{\circ}$. Note: the waveplate $\mathrm{FA}$ at $45^{\circ}$ is equivalent to the $\mathrm{FA}$ at $225^{\circ}$.

\begin{tabular}{|c|c|c|c|c|}
\hline \multirow{2}{*}{$\begin{array}{c}\text { Waveplate } \\
\text { FA }\end{array}$} & \multicolumn{2}{|c|}{$\begin{array}{c}\text { CIV } \\
\Delta=282.9^{\circ}\end{array}$} & \multicolumn{2}{|c|}{$\begin{array}{c}\text { MgII } \\
\Delta=138.2^{\circ}\end{array}$} \\
\hline & $2 * V P$ & $2 * \mathrm{HP}$ & $2 * V P$ & $2 * \mathrm{HP}$ \\
\hline $45^{\circ}$ & $I+0.22 Q+97 V$ & $I-0.22 Q-97 V$ & $I-0.75 Q-0.67 \mathrm{~V}$ & $I+0.75 Q+0.67 \mathrm{~V}$ \\
\hline $135^{\circ}$ & $I+0.22 Q-97 V$ & $I-0.22 Q+97 V$ & $I-0.75 Q+0.67 V$ & $I+0.75 Q-0.67 \mathrm{~V}$ \\
\hline $0^{\circ}$ & $I+Q$ & $I-Q$ & $I+Q$ & $I-Q$ \\
\hline $22.5^{\circ}$ & $I+.63 Q+.39 U+.68 V$ & $I-.63 Q-.39 U-.68 \mathrm{~V}$ & $I+.16 Q+.87 U-.46 V$ & $I-.16 Q-.87 U+.46 V$ \\
\hline $67.5^{\circ}$ & $\mathrm{I}+.60-.39 \mathrm{U}+.70 \mathrm{~V}$ & $I-.60 Q+.39 U-.70 \mathrm{~V}$ & $I+.10 Q-.87 U-.48 \mathrm{~V}$ & $I-.10 Q+.87 U+.48 V$ \\
\hline $\mathbf{9 0}^{\circ}$ & $I+Q$ & $I-Q$ & $I+Q$ & $I-Q$ \\
\hline $112.5^{\circ}$ & $I+.63 Q+.39 U-.68 \mathrm{~V}$ & $I-.63 Q-.39 U+.68 V$ & $I+.16 Q+.87 U+.46 V$ & $I-.16 Q-.87 U-.46 V$ \\
\hline $157.5^{\circ}$ & $I+.60 Q-.39 U-.70 \mathrm{~V}$ & $I-.6 Q+.39 U+.70 V$ & $I+.10 Q-.87 U+.48 V$ & $I-.10 Q+.87 U-.48 V$ \\
\hline
\end{tabular}

polarization measurements and how the pointing system $(\$ 2.2)$ reduced the polarization resolution by introducing image motion in our polarization measurements.

\subsection{Waveplate failure}

Table 2 shows measurements that were made during the flight of SUMI. At altitudes above 200km, the mission would concentrate on CIV circular polarization [V] measurements with the waveplate fast axis rotating between $45^{\circ} / 225^{\circ}$ and $135^{\circ} / 315^{\circ}$ positions (in red). Under $200 \mathrm{~km}$ where the CIV lines are weak, SUMI concentrates on the linear polarization measurements $[Q \& \boldsymbol{U}]$. With that in mind, the waveplate fast axis position (FA) at launch is $0^{\circ}$.

During the launch the waveplate was suppose to start calibrations at $100 \mathrm{~km}$. Unfortunately, the waveplate bound up during this phase creating a timing problem between the data system and the waveplate controller which was not observed in our ground-based testing. With this timing error, the waveplate failed to rotate limiting SUMI to linear polarization measurements $\left(\mathrm{FA}=0^{\circ}\right.$, blue in Table 3). The waveplate timing error also reduced the number of images that we expected to acquired due to a time out condition where the cameras were waiting for the waveplate to reach its programmed position. For example, SUMI obtained $961 \mathrm{MgII}$ (VP6\&HP3) images but we expected more than 1500 images during our 6 minute flight.

After SUMI was returned from WSMR, extensive testing of the waveplate was done to determine the source for this failure. The failure was determined to be a timing error between the command to move and a polling request command. While a one microsecond delay in these commands was been shown to resolve this failure, a one millisecond delay will be added to all of the microcontroller commands to eliminate this problem in future flights.

\subsection{Pointing system problems}

During the flight, the pointing problem was not obvious in the realtime images from our slit jaw camera. Only after the flight when a movie was made of the slitjaw images was the problem obvious.

Figure 6 shows the random nature of the image motion. The telemetry (roll, pitch and yaw) from the SPARCS pointing indicated that the pointing stability was better than 0.5 " while the image motion seen by the slitjaw camera was two orders of magnitude larger. Analysis of the slitjaw motion and the changes in the gas pressure that controls the pointing indicated a direct relationship between the two. Since the slit did not move in the slitjaw images, that left the SUMI telescope or some "unknown" anomaly in the SPARCs pointing system. After a careful examination of the SPARC's data, the SUMI telescope became the leading candidate for the image motion.

While a failure in the epoxy joints holding the primary mirror to its holder might explain the image motion, the primary mirror holder had no tilt or alignment adjustments. All of the adjustments for the SUMI telescope are on the secondary 


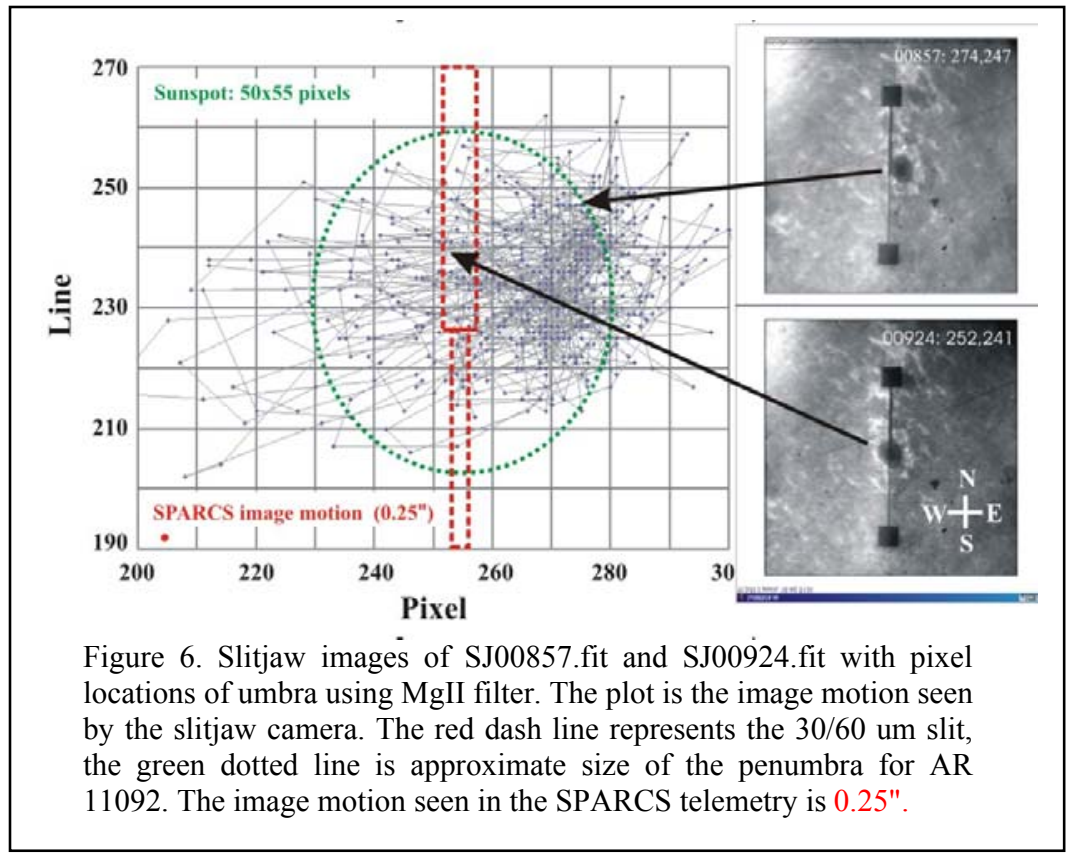

mirror holder. No problems were noted during the inspection of the epoxy joints on both the primary and secondary mirrors. Only after disassembling the secondary spider and checking the secondary tilt mechanism did the source for SUMI's pointing problem become obvious. Although the tilt mechanism was okay, two set screws on the focus mechanism were missing which allowed it to back away from the secondary tilt assembly. Without this support, the secondary was allowed to tilt $\pm 0.05^{\prime}( \pm 1.2$ arc minute in Figure 6).

While the image motion allowed SUMI to observe various structures in AR 11092 (Figure 7), a scientific objective for this first mission is to measure the Stokes polarization from a simple active region. Therefore, stable pointing is required to integrate up enough photoelectrons to get the signal to noise $(\mathrm{S} / \mathrm{N})$ required to detect the polarizations levels that were expected in the Sun's transition region (Figure 14), especially when limited to linear polarization measurements. The consequences of this image motion will be described in greater detail in next section.

\section{DATA ANALYSIS}

One of the scientific goals of SUMI was to obtain the full Stokes polarization [I,Q,U,V] measurements on the MgII emission lines. AR 11092 would have been an ideal candidate for this first flight if the launch could have been delayed 3 days since it was a large simple H-type sunspot that would provide a "potential" magnetic field and allow us to compare photospheric magnetic field measurements with SUMI's chromospheric/transition region measurements. With the failure of the waveplate, SUMI's data was limited to MgII linear polarization measurements ( $\$ 2.1)$.

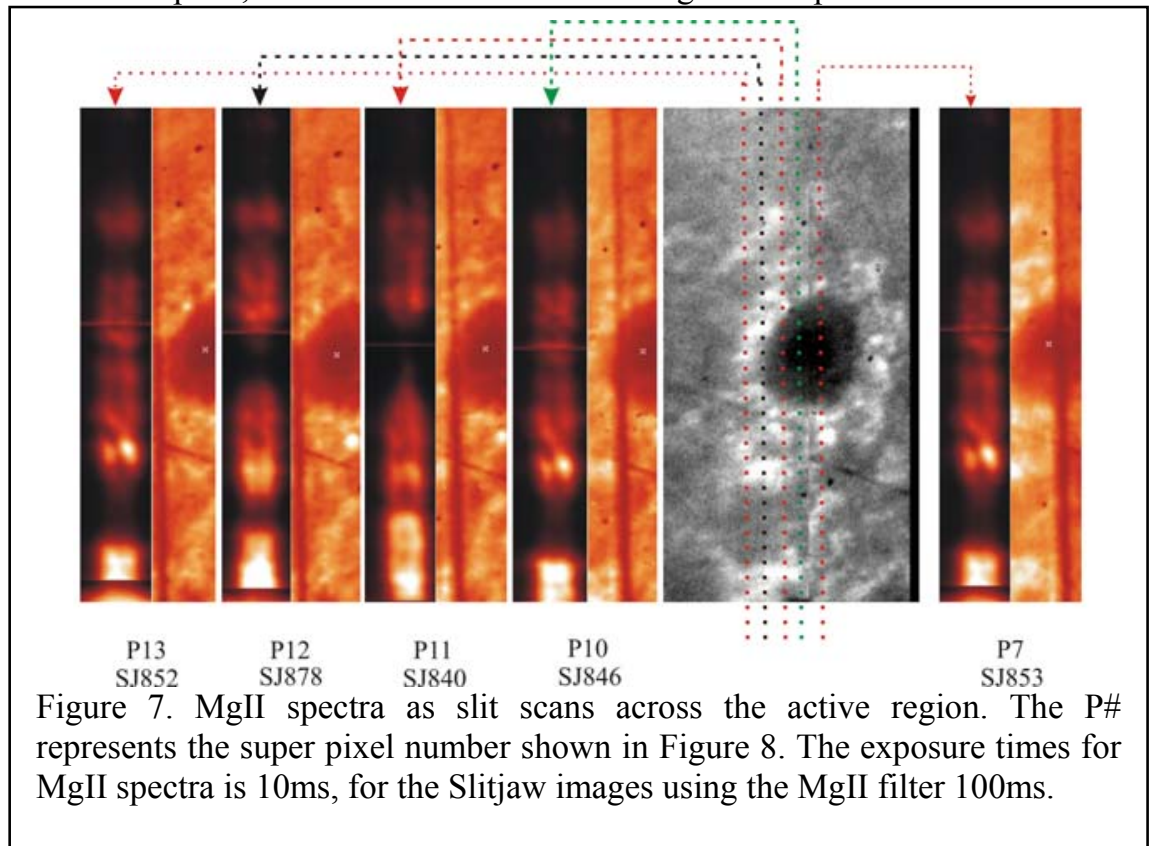

With this simple region (Figure 5), solar activity would be low and intensity crosstalk would not be a problem. Therefore, each polarization channel (VP6 and HP3) could be analyzed independently. Completing the polarization analysis for each channel (VP6 and HP3, see Table 3), the software to correct for the instrumental errors between the two gratings would then be developed. Unfortunately, the waveplate failure limited our ability to calibrate/correct for instrumental errors between the two polarization channels. The expected instrumental errors are: (1) differences in the optical efficiency between the two gratings, (2) the $\mathrm{X} / \mathrm{Y}$ magnification due to the different distances between the slit 
and the gratings, (3) small rotation errors in the images (CCD cameras and gratings) and (4) the aberrations over the field of view for the two gratings (West, et al, 2011a). Of those, the variation of the aberrations/spot size over the field of view of the VP6 and HP3 polarization channels is the most difficult error to correct. While a Zemax optical model can produce an "ideal" model for differences between the two optical paths, the task becomes labor intensive when manufacturing (gratings: rulings, ruling axis versus toroidal axis, toroidal radii and stress induce mounting) and alignment (gratings, fold mirrors, cameras: optical axis position, tilt, and rotation) errors must also be considered.

\subsection{Requirements to obtain $10^{-3}$ linear polarization}

With a region so close to the limb, the linear polarization measurements are expected to be stronger. Unfortunately, the pointing system became the limiting factor in the MgII polarization analysis. In order to improve the signal to noise $(\mathrm{S} / \mathrm{N})$ of the polarization measurements, our earlier radiometry calculation indicated that eight (8) images would be required to get a $\mathrm{S} / \mathrm{N}$ of $\mathbf{4 0 4 8}$ for plage regions and $\mathbf{9 3 2}$ for quiet regions to meet our scientific goals. Eight images is not a large number but it assumes stable pointing $(\S 2.2)$ and exposures optimize for $80 \%$ well capacity for the CCD. Since the exposure times cannot change to get the required $\mathrm{S} / \mathrm{N}$, the number of images must be different for plage and quiet

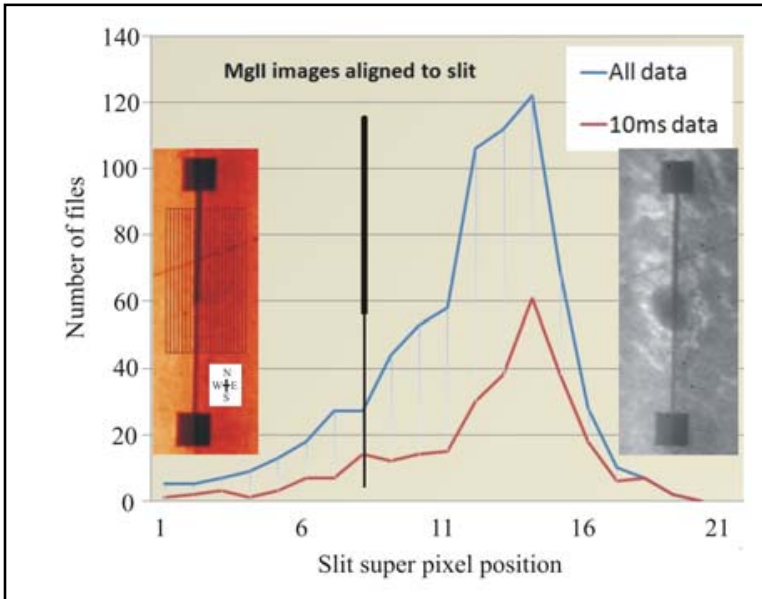

Figure 8. Distribution of MgII images in 5 pixel bins parallel to the slit. Bin 8 is center of sunspot on slit (right image, SJ00723 x/y position 245,230). In SUMI's observations, the sunspot is 20 to $30 \mathrm{SJ}$ pixels (10"-20") east of the slit. regions that are centered onto the slit. Therefore, our post flight goal for the MgII linear polarization measurement is obtain a minimum of 16 images that can be coaligned to achieve our $\mathrm{S} / \mathrm{N}$ requirements.

With the uncertainties in our radiometry calculations, two exposure times were used for the MgII observations: $10 \mathrm{~ms}$ and $100 \mathrm{~ms}$. Both of these exposure times were acceptable for the $30 \mu \mathrm{m}$ slit but when plage was observed in the $60 \mu \mathrm{m}$ slit, the $100 \mathrm{~ms}$ exposures were saturated. Therefore, most of our analysis has been limited to the $10 \mathrm{~ms}$ exposures (lower curve in Figure 8).

In order to obtain enough images, a 5x144 "super-pixel" structure was developed and all of the MgII images with the sunspot in that super-pixel were sorted into a spreadsheet. Figure 8 shows the distribution of the MgII images within that super pixel grid. Comparing Figures 6 and 8, the slit is west of the sunspot in most of SUMI's images (the slit is in super pixel 8, black line in Figure 8). The next section will describe the calibration and alignment of the 10ms MgII images that will be used to produce SUMI's linear polarization measurements.

\subsection{MgII measurements}

SUMI obtain over $500 \mathrm{MgII}$ spectra of AR11092 during its flight. The maximum number of spectra with a $10 \mathrm{~ms}$ exposure time was in super pixel 13 which had 57data sets (Figure 8). Therefore, it is that data set that will be used in our polarization analysis. While the slitjaw images "suggest" that SUMI has enough data sets to achieve our polarization resolution goal $\left(>16\right.$ images and $<10^{-3}$ ), the fine structure of the MgII emission requires additional 

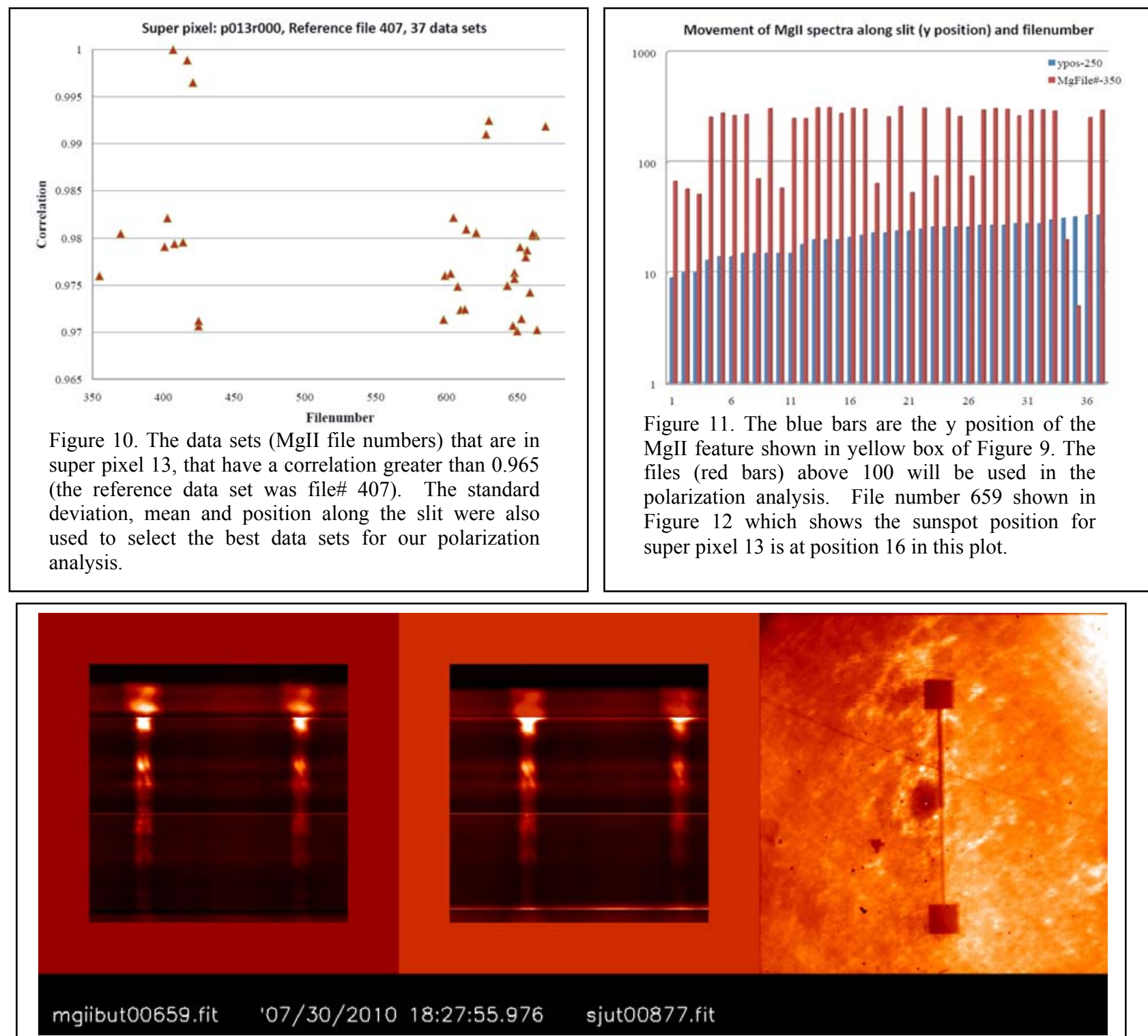

Figure 12. SUMI MgII spectrum with slit jaw image for super pixel 13. Left MgII image is the $\mathrm{HP}=[\mathrm{I}-\mathrm{Q}]$ and the right image $\mathrm{VP}=[\mathrm{I}+\mathrm{Q}]$. The slit jaw image was obtained using the narrowband MgII filter. NOTE: orientation is the same as the HMI image in Figure 13.

processing to minimize the image motion effects in this data set. Figure 9 shows three data sets that were placed in super pixel 13 and the image motion along the slit.

While slit jaw images and MgII spectra may be close in time, the effects of image motion appear to be larger in the spectra due to the limited field of view of the slit. Therefore an additional step in the MgII analysis is to minimize the image motion effects by doing a correlation analysis of all of the files in a super pixel. The yellow box in Figure 9 (file\# 417) is the MgII feature that was used to further reduce our data set. The only data sets that are used in the polarization analysis are shown in Figure 10.

In Figure 10, there are two distinct data sets. The first data set is early in the flight when there was a lot of image motion (files 350-430) and the second data set when the pointing system was becoming "stable" (files 580-670). Therefore, our analysis will use the second data set in our MgII linear polarization measurements. 
Figure 11 shows the same data set as Figure 10 but has the data sorted with the movement along the slit (Y pixel position -250). Of the 37 data sets that met our correlation requirements (Figure $10)$, we are left with 26 data sets.

\subsection{Optimizing the MgII data}

While super pixel 13 has the most data sets, and hence the best chance for measuring linear polarization in MgII, this location is not ideal. Figure 12 shows the MgII spectrum (file\# 659) for super pixel 13 and the corresponding MgII slitjaw image. Figure 13 shows the HMI photospheric measurements of the magnetic field. The HMI data was rotated to match the SUMI slitjaw images and the linear polarization measurements to match SUMI's analyzer. Since this region is nearly "potential", the magnetic structure that is observed in the photosphere is "expected" to be similar to the structure observed at the MgII height. Except in sunspots, the strongest linear polarization signal is $\boldsymbol{- U}$ (the polarization direction that is toward the limb of the Sun).

\subsection{MgII polarization analysis}

While we have identified the necessary number of data sets that can be processed to achieve our $10^{-3}$ polarization resolution, most of those data sets are late in the mission when the pointing system/secondary had "stabilized". At that time our slit, unfortunately, ended up on the west side of the active region where linear polarization is expected to be weak. Therefore, reducing the instrumental errors to $<10^{-4}$ will be required.

Some of the instrumental errors are not difficult to correct. The difference in the optical efficiencies of the two beams (HP3 and VP6) which includes the difference in the polarization reflectivity of the gratings ${ }^{13}$ and the electronic gain of the two cameras is a simple correction. There are two magnification corrections that must be made. In order to minimize the mechanical contact between the gratings during launch, the gratings are offset in the z-axis. Therefore, HP3 is closer to the telescope focus and has a slightly larger magnification at the detector than VP6. Since the gratings are toroidal, this magnification is different in the $\mathrm{X}$ and $\mathrm{Y}$ axes. To transform the HP3 images to the VP6 scale we must multiply the HP3 array size by $0.9578( \pm 0.0037)$ in the $X$ axis and $0.9672( \pm 0.0032)$ in the $\mathrm{Y}$ axis.

For the MgII images, image rotation can be caused by the gratings or the cameras. While the source of this error is not important, determining this rotation angle has been difficult and will require looking at data not include in our super-binned data sets. Our current estimate for the rotation between the VP6 and HP3 images is $-0.539 \pm 0.442$. While the error "looks" small, the $\mathrm{MgII}$ lines are near the edge of images where the rotation could be as large as 5 pixels. A program is being developed to automate the analysis of this error and will include all of our $10 \mathrm{~ms}$ images. We hope this will reduce the large uncertainty in the rotation angle.

The resolution difference between the two optical paths has not been quantified. While our Zemax model can show the resolution errors, it assumes that the grating alignment survived the launch (and landing). After the magnification and rotation

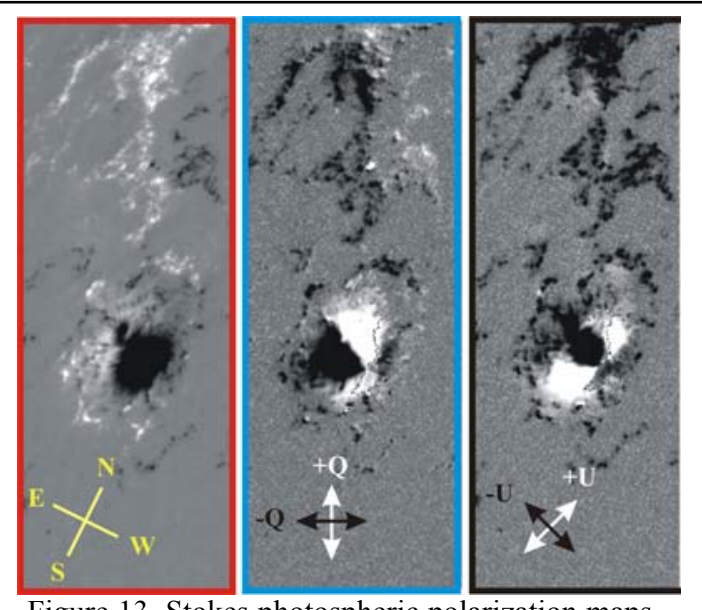

Figure 13. Stokes photospheric polarization maps obtain from the HMI vector magnetic field measurements on the Solar Dynamics Observatory observed on 7/30/2011 at 1800UT. The polarization images (V/I left image/red box and $\mathrm{Q} / \mathrm{I}$ center/blue box) images were rotated to match the SUMI slit jaw images and the HMI linear polarizations ( $\mathrm{Q}$ and $\mathrm{U}$ ) were rotated to the same coordinate system as the SUMI analyzer $(+/-\mathrm{Q}=\mathrm{VP}-\mathrm{HP})$. The only transition region polarization measurement made by SUMI is the Q polarization (see \$2.1). The E/W orientation is not the same as Figure 6 and 8.

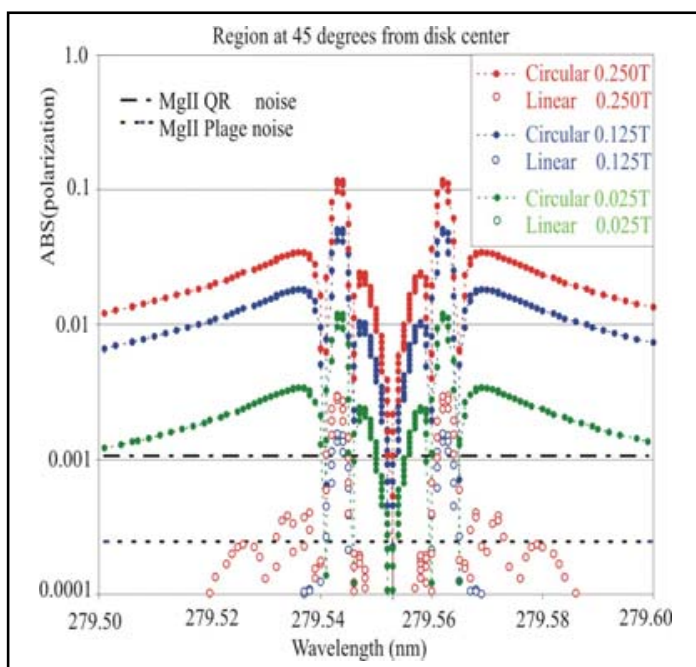

Figure 14. Total linear and circular polarization expected for magnetic fields $\left(\mathrm{T}=10^{4} \mathrm{G}\right)$ that vary with wavelength $(\mathrm{nm})$. The expected noise levels for three magnetic field strengths for the MgII measurements are indicated by horizontal dash lines. The radiative transfer model was developed by Dr. Han Uitenbrock/NSO for SUMI (private communication). 
errors have been corrected, the current approach will be to analyze the VP6 and HP3 independently as the image of a superpixel data set moves along the slit (Figure 11). Assuming that the image motion has been eliminated (wavelength axis), a Zemax model can be developed to match the observed change in resolution. If successful, this final correction might lead to a real solar linear polarization measurement.

\section{SUMMARY}

The failure of the waveplate limited us to only MgII linear polarization measurements. While the active region that we observed on 7/30/2010 was not ideal for full Stokes polarization measurements (preferred location of active region: near the center of the disk within one half of the solar diameter), the region would have been ideal for linear polarization measurements. Unfortunately, the image motion and the "final" position of the slit has made the linear polarization analysis difficult.

Although the probability of reducing SUMI's instrumental errors below our goal of $10^{-4}$ and obtaining a "real" solar MgII linear polarization signal is low for this initial engineering flight, we will continue to develop our scientific software since this research will be important in the data analysis of SUMI's next flight which is scheduled for the summer of 2012.

\section{REFERENCES}

[1] Gary, G. A., "Plasma Beta above a Solar Active Region: Rethinking the Paradigm," Solar Phys., 203, 71 (2001).

[2] Rosner, R., Low, B. C., and Holzer, T. E., "Physical processes in the solar corona," [Physics of the sun], D. Reidel Publishing Co, 135-180 (1986).

[3] Moore, Davis, and Hathaway, "High-Resolution Solar Magnetography from Space: Beyond Solar-B," http://solarscience.msfc.nasa.gov/Beyond Solar-B.shtml, (2001)

[4] Davis, J., West, E., Moore, R., Gary, G., Kobayashi, K., Oberright, J., Evans, D., Wood, H., Saba, J. L. R., and Alexander, D., "MTRAP: The Magnetic Transition Region Probe," Proc. SPIE 5901, 273-280 (2005).

[5] Gurman, J. B., "The MG II H line in sunspot umbrae," Solar Phys., 90, 13-15 (1984).

[6] Uitenbroek, H., "Multilevel Radiative Transfer with Partial Frequency Redistribution," Astrophysical Journal, 557, 389-398 (2001).

[7] Peter, H., "On the nature of the transition region from the chromosphere to the corona of the Sun," A\&A, 374, $1108-1120$ (2001).

[8] West, E. A., Porter, J. G., Davis, J. M., Gary, G. A., Rabin, D. M., Thomas, R. J., and Davila, J. M., “Overview of the Solar Ultraviolet Magnetograph Investigation," Proc. SPIE 4139, 350-361 (2000).

[9] West, E. A., Porter, J. G., Davis, J. M., Gary, G. A. and Adams, M., "Development of a polarimeter for magnetic field measurements in the ultraviolet," Proc. SPIE 4481, 109-117 (2001).

[10] West, E. A., Kobayashi, K., Davis, J. M., and Gary, G. A., "The Solar Ultraviolet Magnetograph Investigation Sounding Rocket Program,” Proc. SPIE 6689, 66890E-12 (2007).

[11] Kobayashi, K., West, E. A. and Noble, M., "Polarization measurements in the Vacuum Ultraviolet," Proc. SPIE 5888, 1-12 (2005).

[12] West, E. A., Porter, J. G., Davis, J. M., Gary, G. A., Kobayashi, K. and Noble, M., "The Solar Ultraviolet Magnetograph Investigation: Polarization Properties," Proc. SPIE 5901, 226-235 (2005).

[13] Kobayashi, K., West, E. A., Davis, J. M. and Gary, G. A., "Polarization measurements on SUMI's TVLS gratings", Proc. SPIE 6682, 1-12 (2007).

[14] Thomas, R. J., “Toroidal varied line-space (TVLS) gratings, ” Proc. SPIE 4853, 411-418 (2003).

[15] Poletto, L. and Thomas, R. J., "Stigmatic Spectrometers for Extended Sources: Design with Toroidal Varied Line-Space (TVLS) Gratings, ” Applied Optics, 43, 2029-2038 (2004). 


\section{Mgll observations using the MSFC Solar Ultraviolet Magnetograph (SUMI)}

Edward West ${ }^{a}$, Jonathan Cirtain ${ }^{a}$, Ken Kobayashi ${ }^{b}$, John Davis ${ }^{b}$ and Allen Gary ${ }^{b}$

- Science goals

- Description of instrument

- Description of flight $(7 / 30 / 2010)$

- Problems during flight

- Mgll measurements

- Summary 


\section{Beta $(\beta)$ plot}

\section{$\beta=$ ratio of gas pressure}

to magnetic pressure

- Photospheric vector magnetic fields only show small changes with large flares

- Goal: to observe changes in the transition region magnetic field where the magnetic field dominates

- Mgll at 280nm (> 40km)

- CIV at $155 \mathrm{~nm} \quad(>200 \mathrm{~km})$

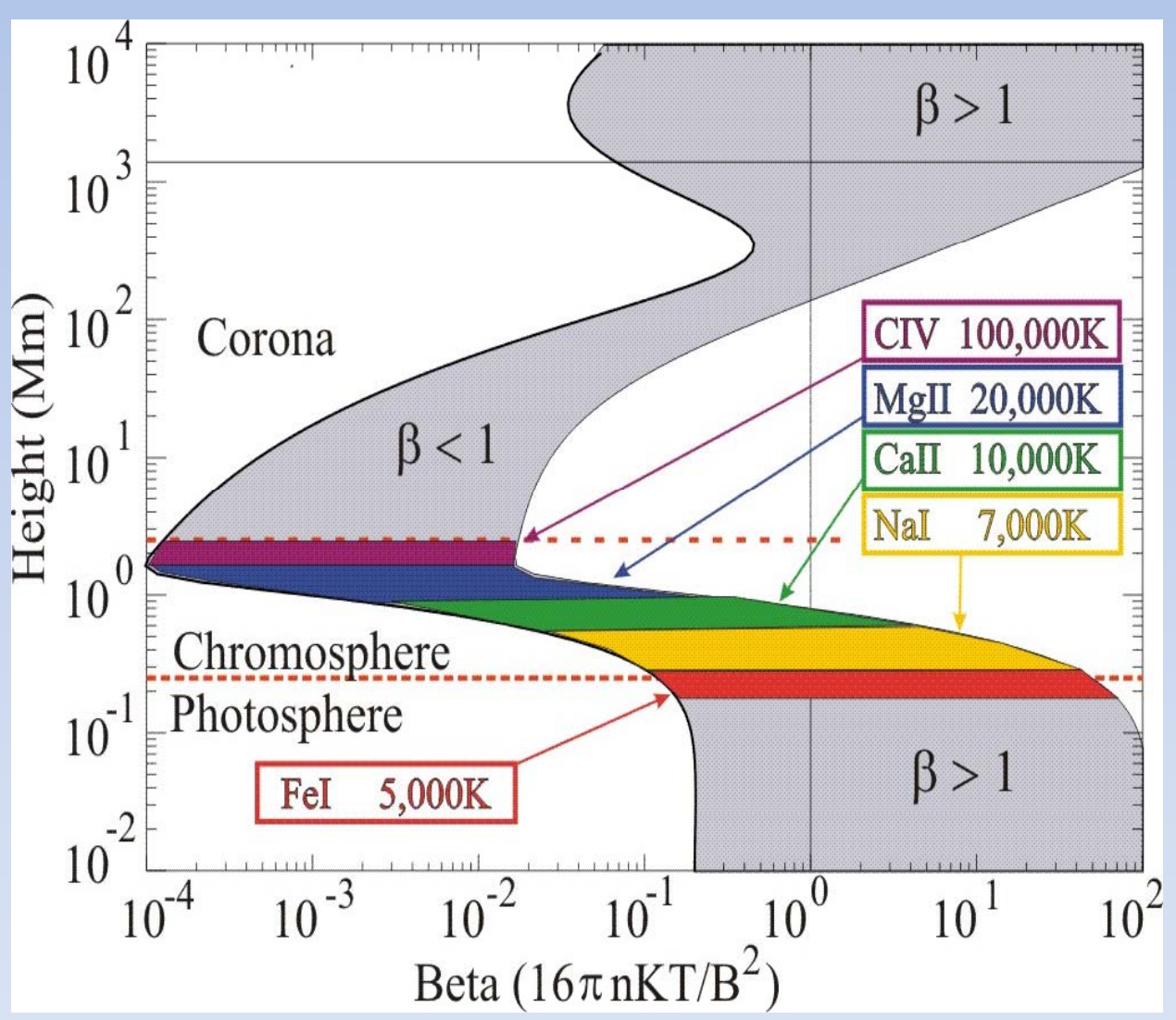




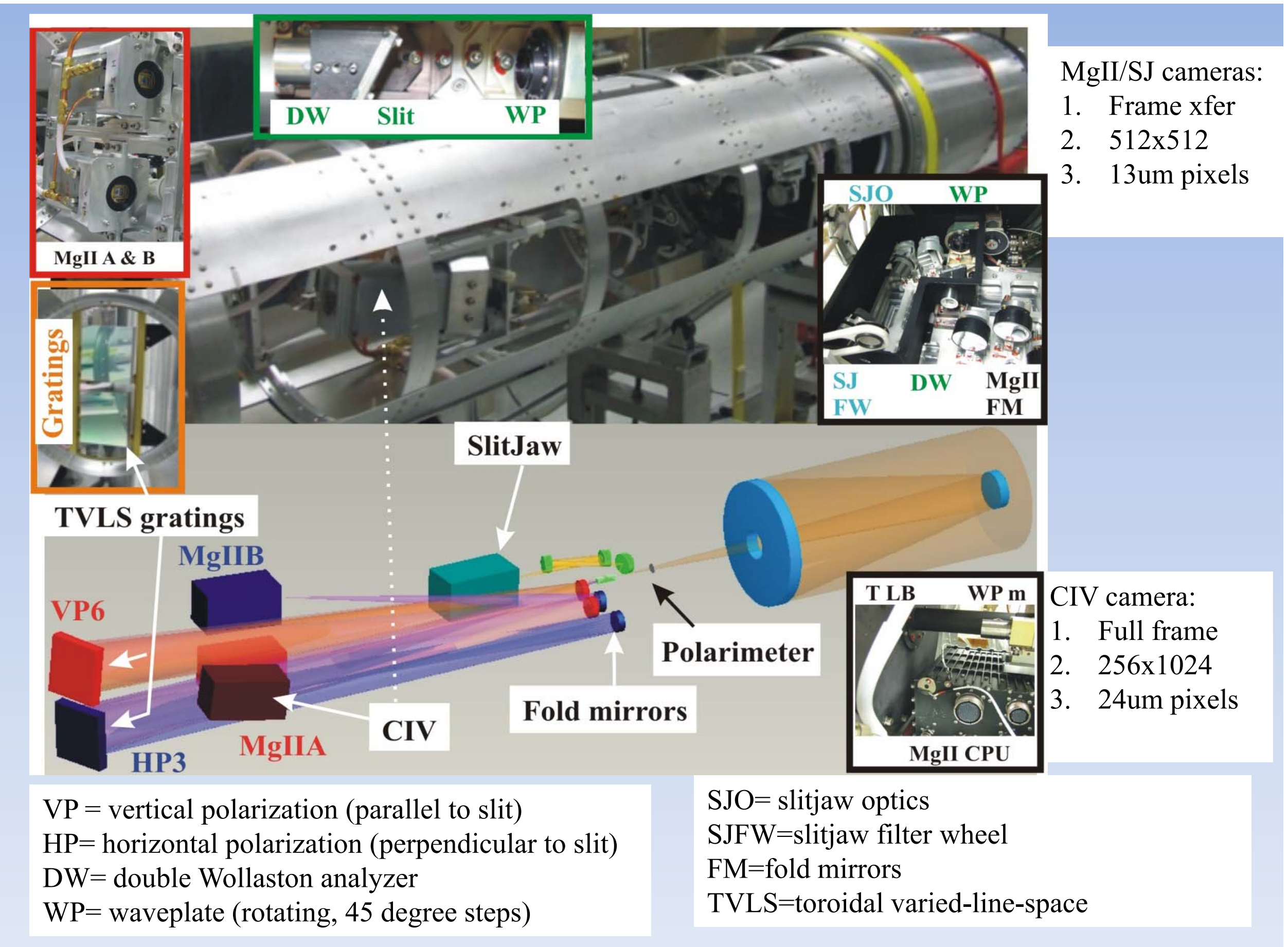




\section{SUMI launch: 7/30/2011 (Friday)}

SUMI met all of its success criteria

- Successful launch and landing

- Acquired target

- Obtain data from all of the scientific cameras

- Verify exposure times and radiometry

- Verify that the alignment of optics survived launch

- Successfully saved and retrieved all of the images from the scientific cameras to the onboard disk drives

Modify SUMI's launch criteria:

- Need a simple "potential" active region within 0.5 solar diameter of disk center

- No launch window the following week

- No guarantee of an active region on the Sun in two weeks (long solar minimum) 


\section{AR11092 observed on $7 / 30 / 2010$}

(HMI images with SUMI Mgll slitjaw image)

1. Active region did not meet our launch criteria: within 0.5 solar radius of disk center

2. Two week delay in launch AND no guarenttee of another sunspot

3. First mission and region would allow us to evaluate engineering performance
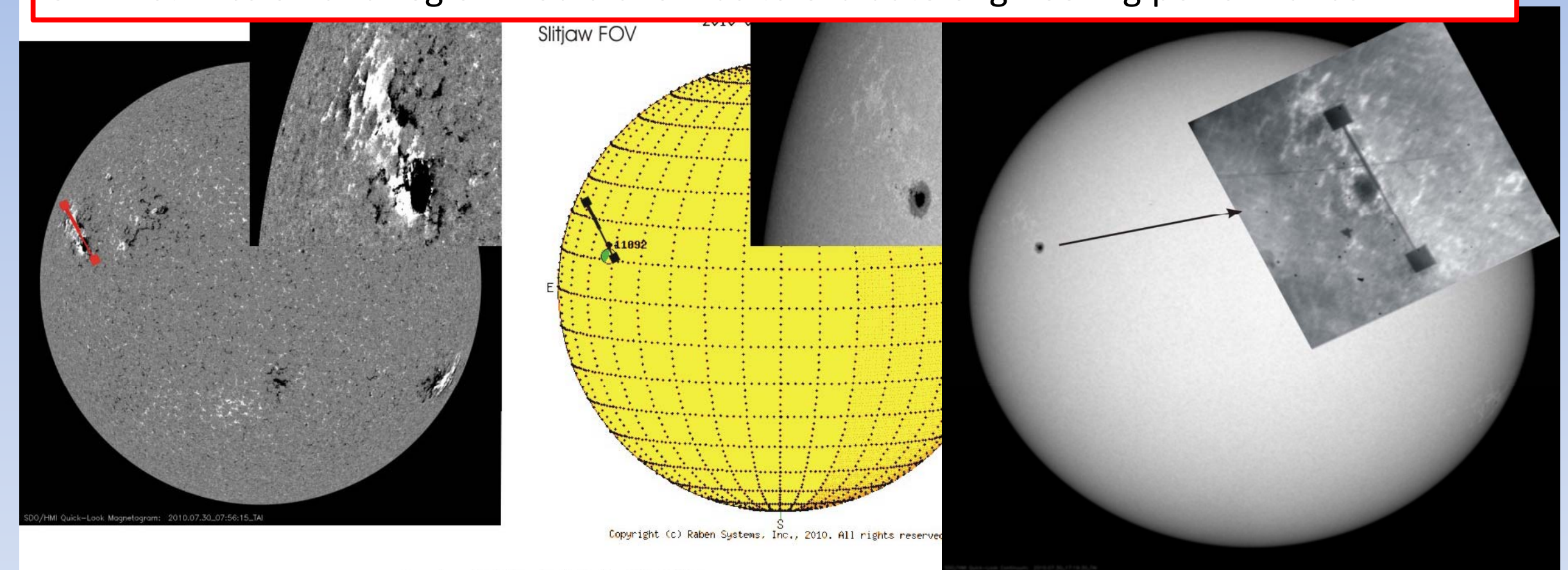

Region 1092 at 1900UT N14E53

Two problems occurred during flight:

1. Waveplate failed to rotate (bound up during launch causing timing error)

2. Mechanical stop on secondary mirror moved $\rightarrow$ allowed secondary to tilt with pointing control 


\section{Waveplate failure: Fast Axis at $0^{\circ}$}

Table 1. Polarization Response to SUMI's rotating waveplate ( $\Delta=$ measured retardance, Kobayashi, 2005). With the waveplate rotation failure, the only measurements that were made were with the fast axis (FA) at $0^{\circ}$. Note: the waveplate FA at $45^{\circ}$ is equivalent to FA at $225^{\circ}$ in Table 3.

Waveplate CIV $\Delta=282.9^{\circ}$

$\operatorname{MgII} \Delta=138.2^{\circ}$

FA

$2 * V P$

$2 * \mathrm{HP}$

$2 * \mathrm{VP}$

$2 * \mathrm{HP}$

$45^{\circ}$

Eliminated CIV circular

$135^{\circ}$ polarization measurements

$0^{\circ}$

$22.5^{\circ}$

$67.5^{\circ}$

$90^{\circ}$

CIV linear signal to weak for sounding rocket flight

No measurements

$112.5^{\circ}$

$157.5^{\circ}$

CIV linear signal to weak for
sounding rocket flight

$I+Q$

I-Q

No measurements 


\section{Mgll images versus slit position near sunpot}

SUMI scanning the active region

BUT purpose was to make polarization measurements.

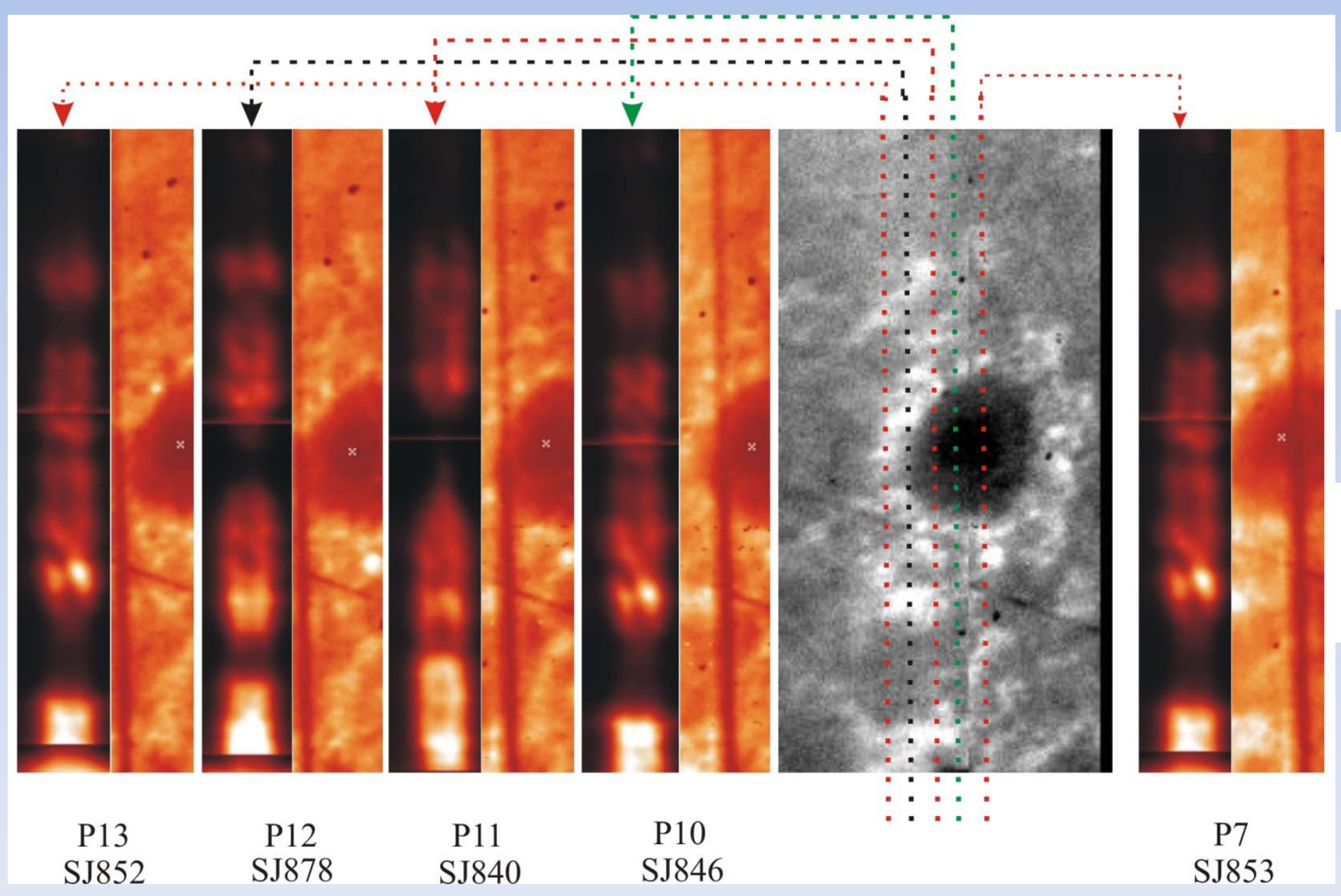

Slitjaw images with Mgll "narrowband" filter inserted

Exposure times:

Mgll spectra $=10 \mathrm{~ms}$

$\mathrm{Mg} \| \mathrm{SJ}=100 \mathrm{~ms}$ 


\section{Image motion seen by slitjaw camera}

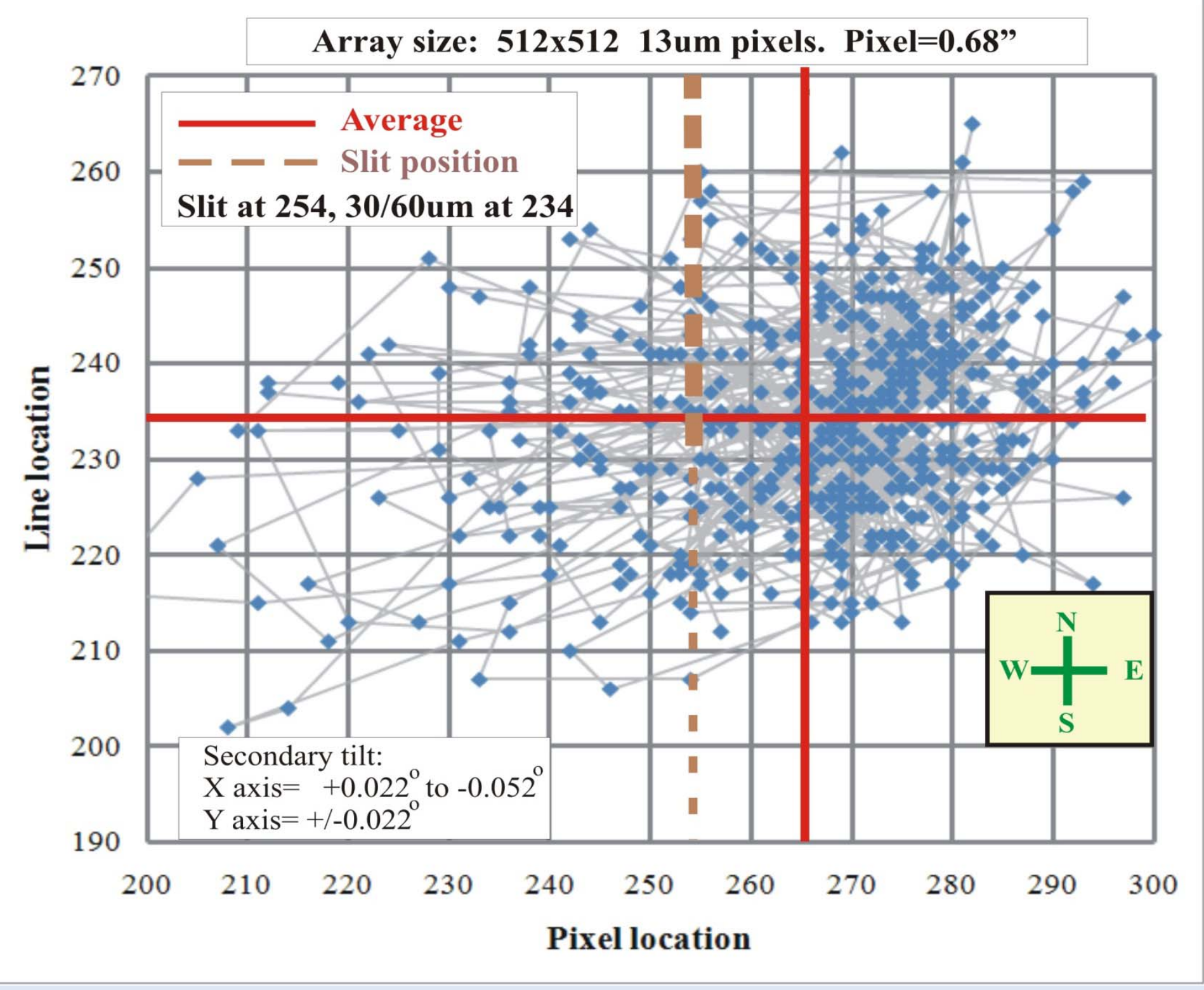




\section{Requirements for MgII linear polarization measurements}

- $\mathrm{S} / \mathrm{N}>1000 / 1$ (next slide)

- To obtain $\mathrm{S} / \mathrm{N} \rightarrow>1,000,000$ photons

- Assumes system photon noise limited

- Assuming MgII camera operating at $80 \%$ well depth

- $\sim 80,000$ photoelectrons/image

- $\rightarrow$ a minimum of 13 images to reach $\mathrm{S} / \mathrm{N}$

- goal to add 16 polarization images 


\section{MgII polarization model}

\section{(Han Uitenbrock/NSO)}

\section{$0.125 \mathrm{~T}=1250$ gauss}
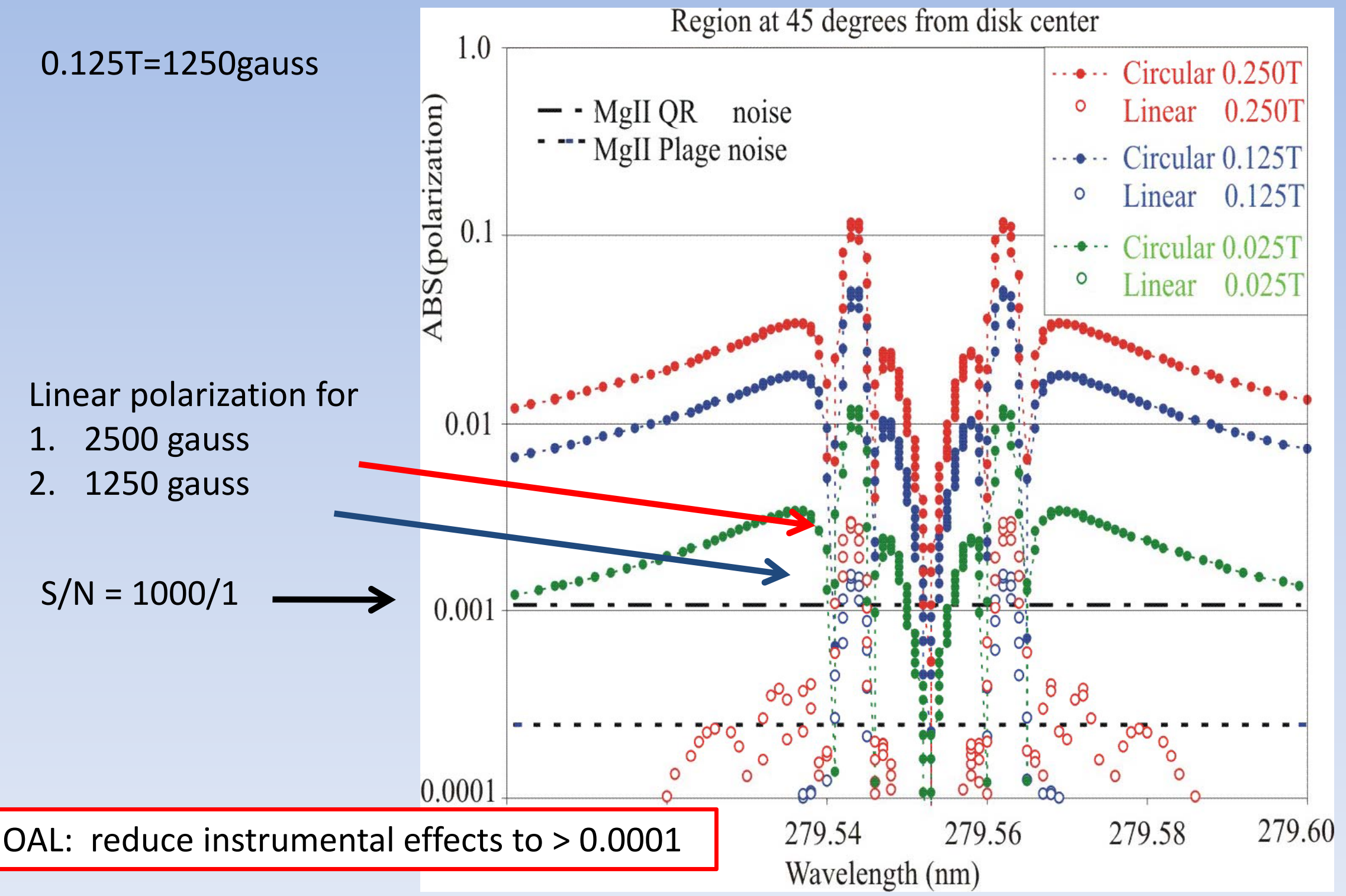


\section{Slitjaw movie of image motion}

Important points:

- Need 16 MgII images for polarization calculation

- The image motion "appears" to be smaller at end of flight

- Slitjaw exposure times:

- $10 \mathrm{~ms}$ with broadband filter

- $100 \mathrm{~ms}$ with MgII filter

- MgII exposure times:

- $10 \mathrm{~ms}$

- $100 \mathrm{~ms}$ (60um slit saturated)

- Slitjaw pixels binned 2x2

- Initial images 256x256 for faster telemetry during pointing stage

- Origin $(0,0)$ top left on images

- Active Region at: N14E53

- North down in images

- North up in motion plot

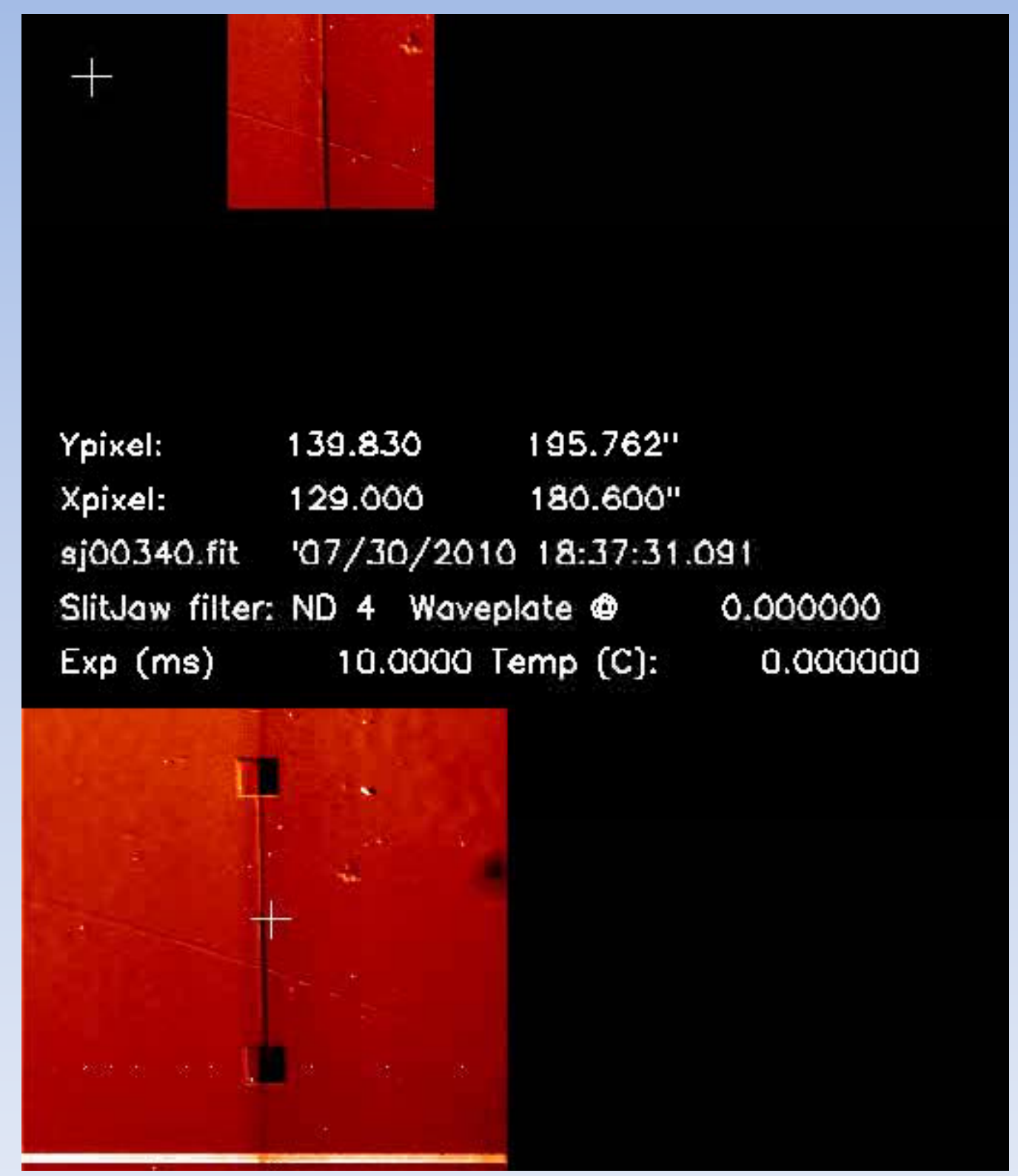




\section{Find 16 images to add for polarization analysis?}

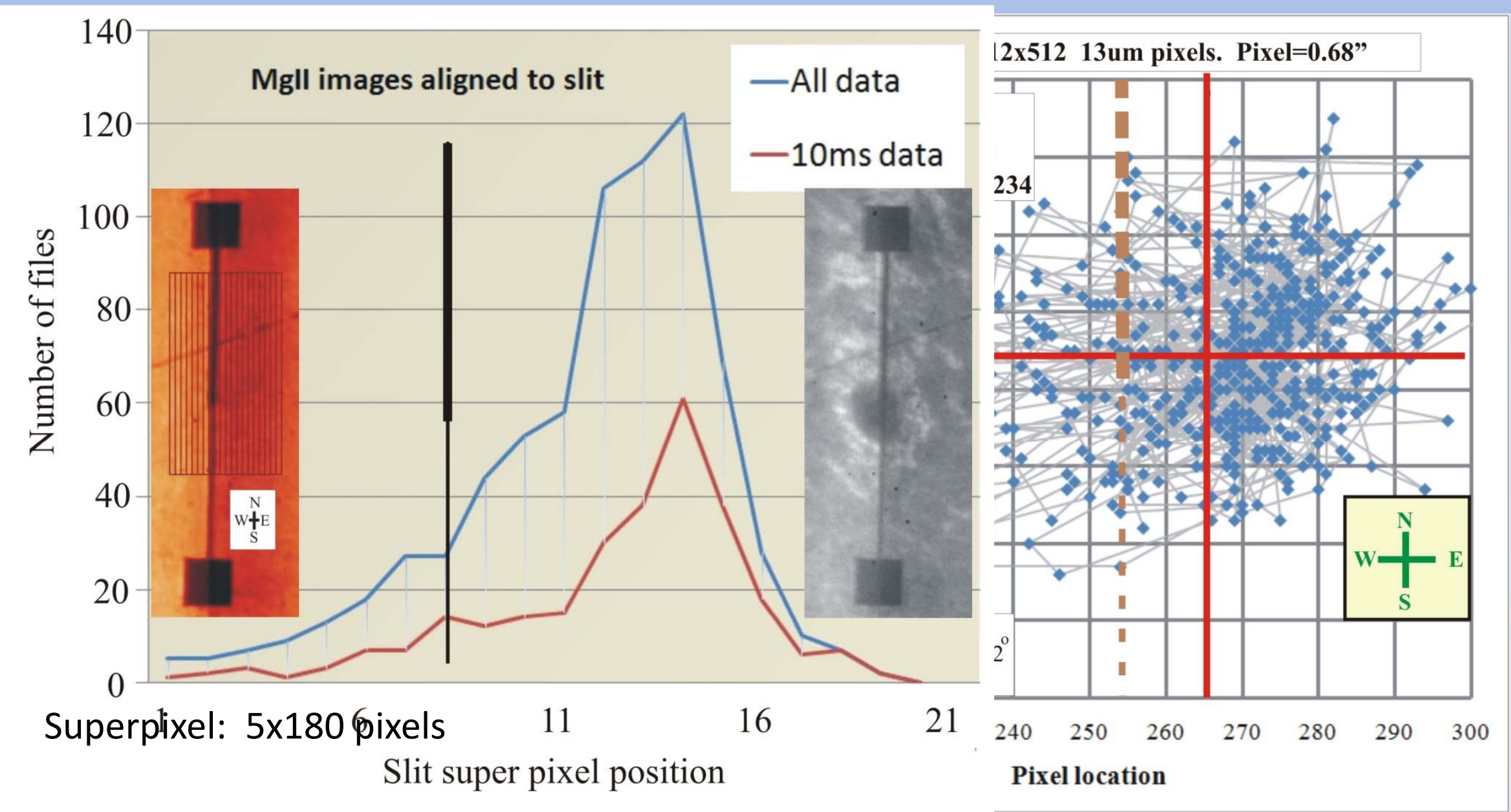




\section{Summary \#1}

HMI polarization maps rotated to align to SUMI slit. The HMI linear measurements were transformed to match the SUMI VP (+Q) and HP $(-Q)$ polarization measurements
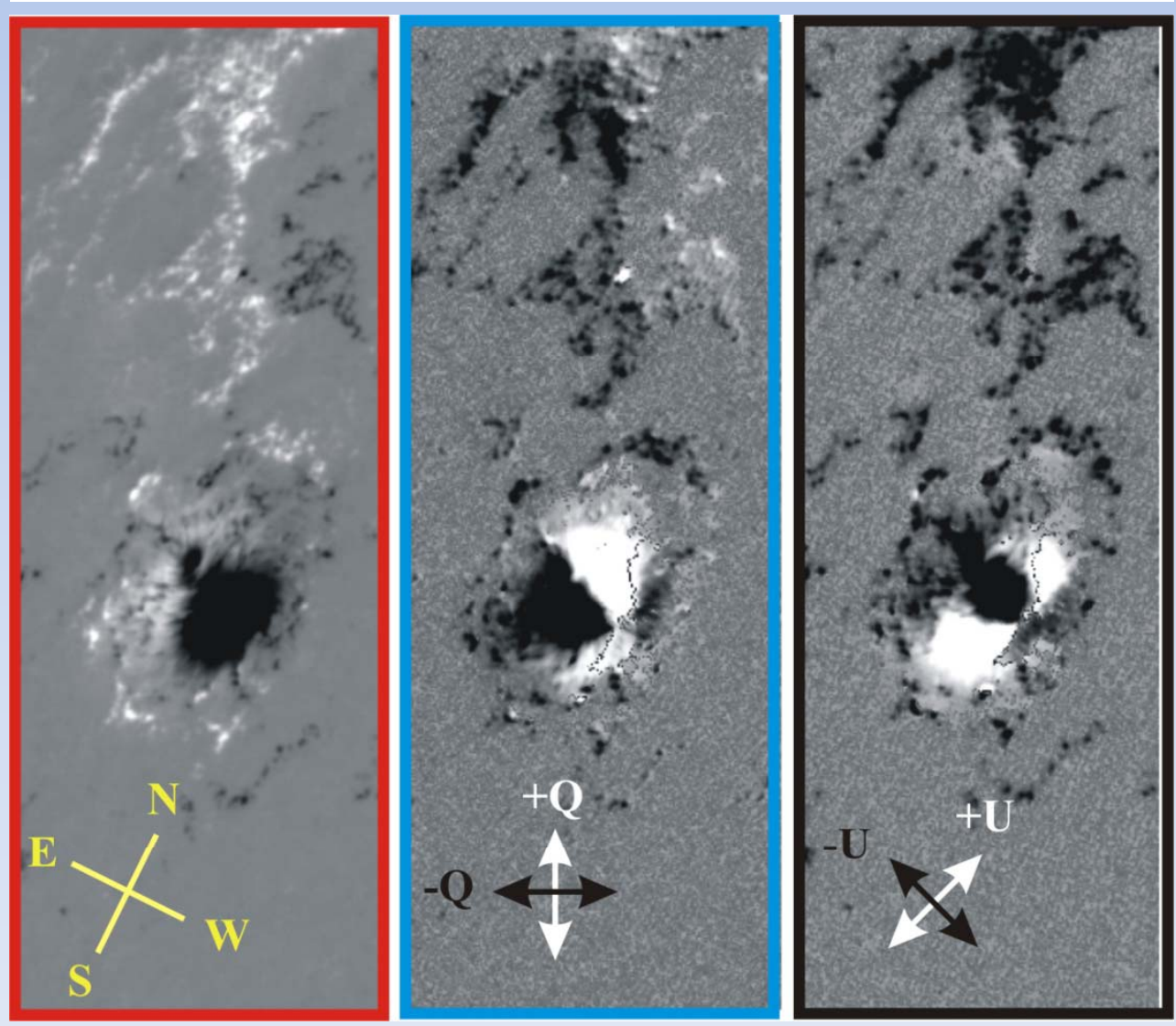

To Be Done:

- Eliminate differences in optical efficiencies between HP3 and VP6 channels

- Eliminate magnification differences

- Different distance from slit

- Different $X / Y$ magnification from toroidal grating

- $X=0.9578 \pm 0.0037$

- $Y=0.9672 \pm 0.0032$

- Eliminate image rotation (camera or grating)

- $-0.539^{\circ} \pm 0.442^{\circ}$

- Correct for aberration differences along slit 


\section{Summary \#2}

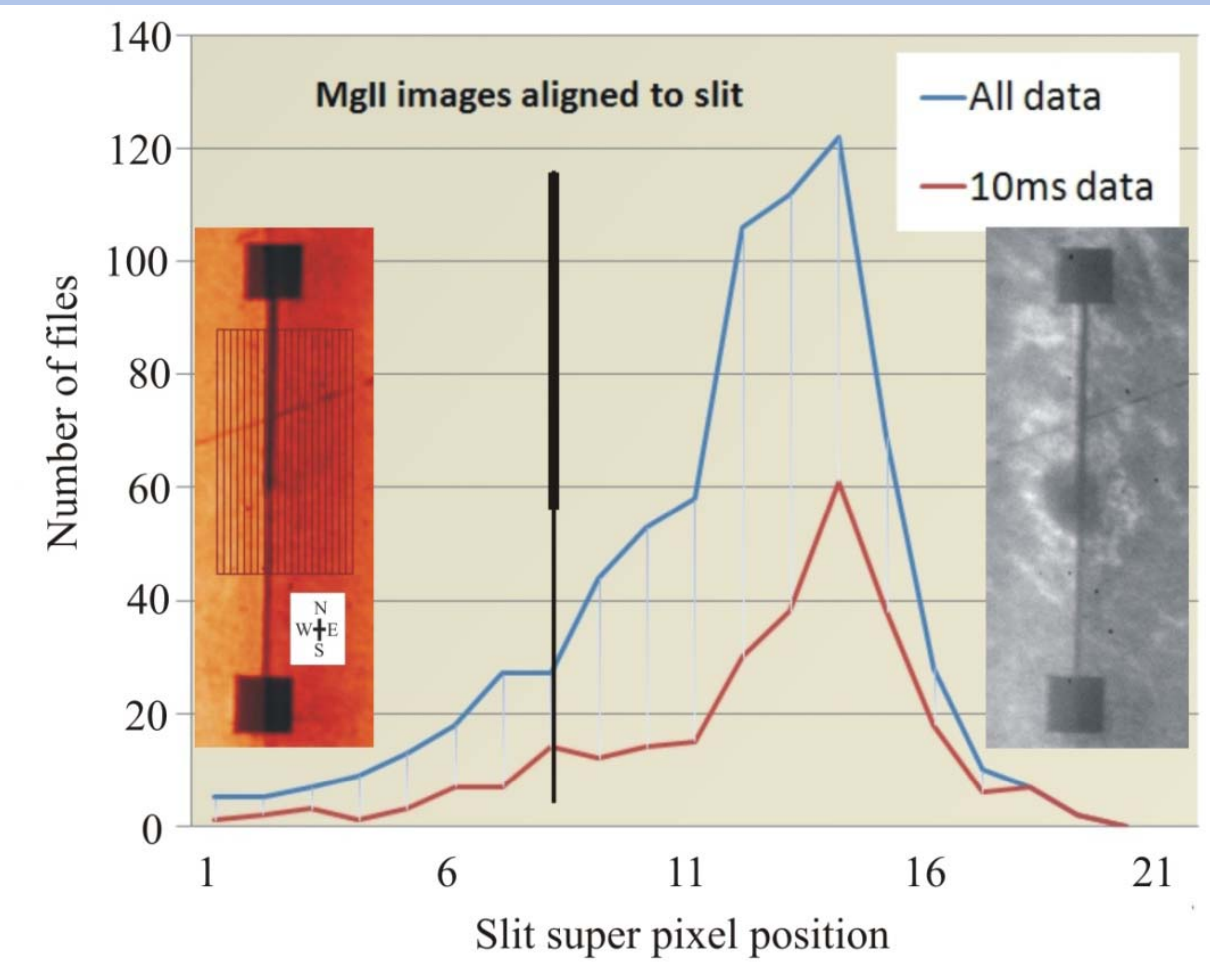

- Goals for software development

- To reduce the instrumental errors below $10^{-4}$

- To be used in reflight mission scheduled for Summer 2012 
Place supporting viewgraphs here for question and answers 

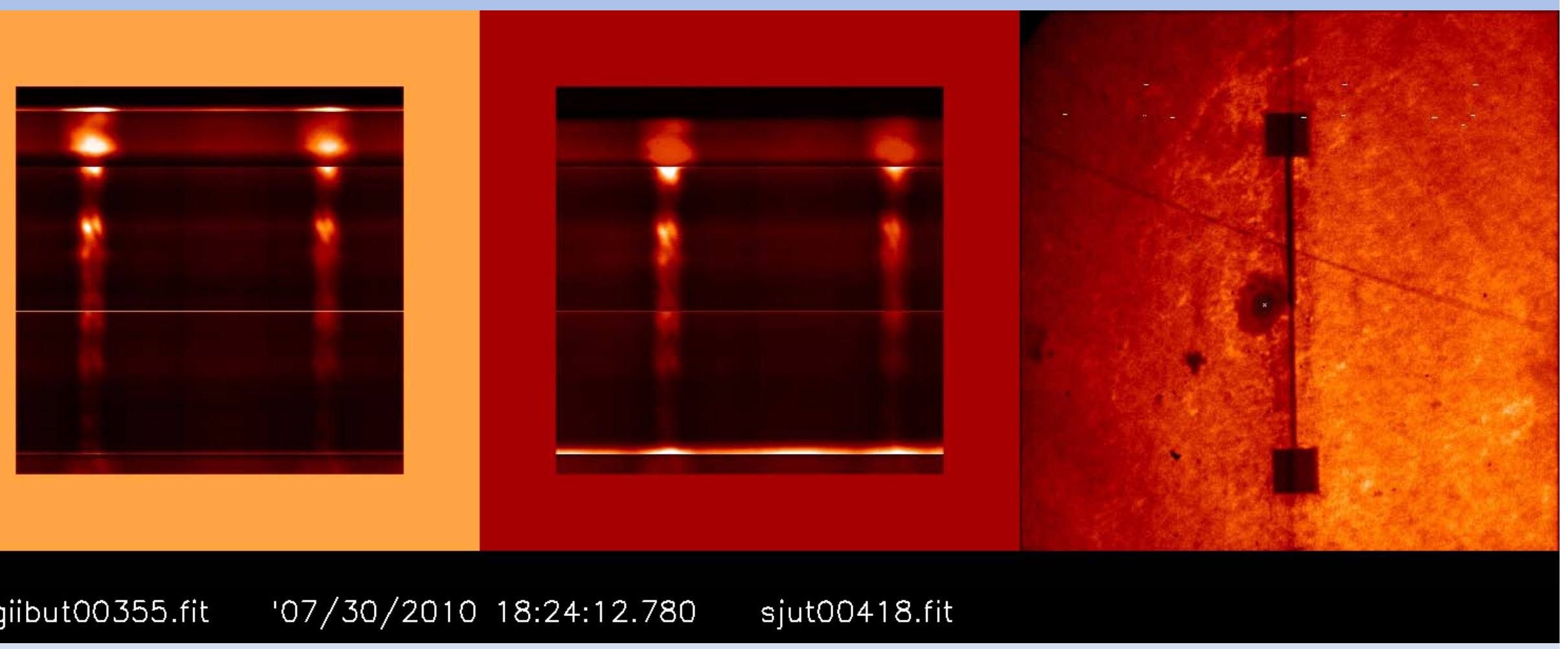

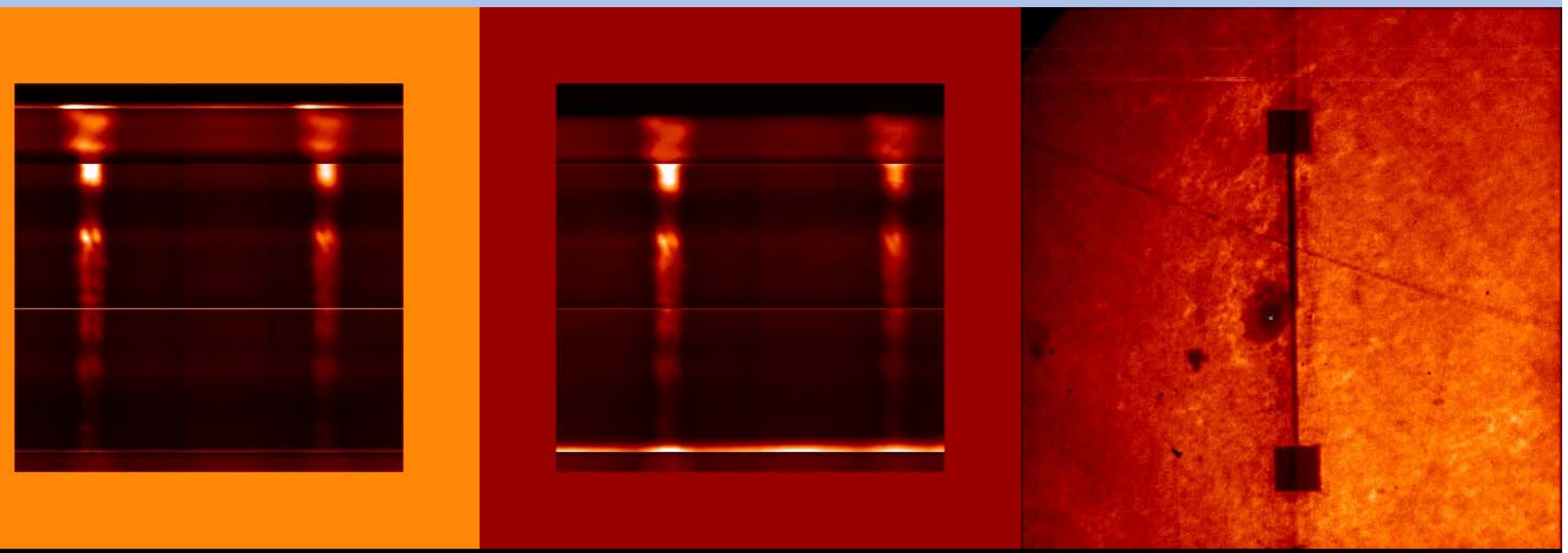

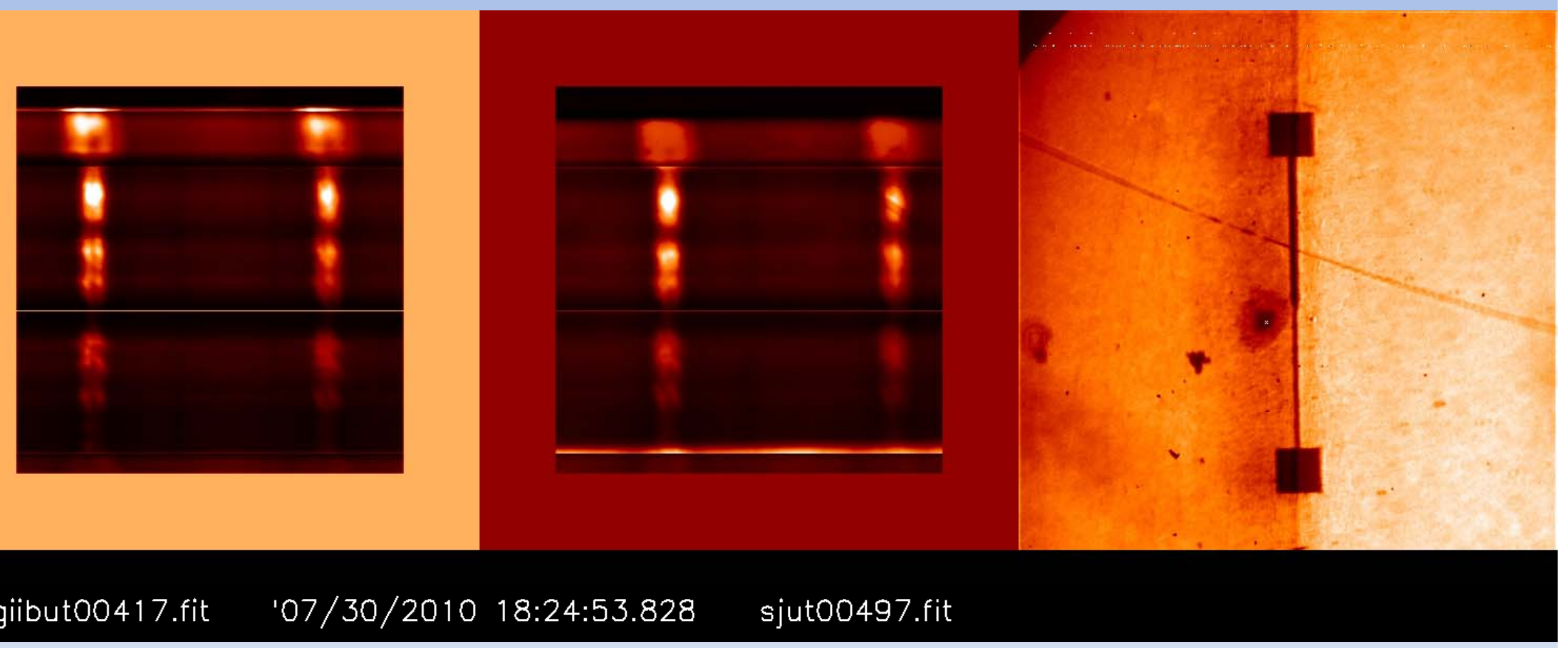